\title{
A GENERALIZATION OF THEOREMS OF FALTINGS AND THUE-SIEGEL-ROTH-WIRSING
}

\author{
PAUL VOJTA
}

In 1929 Siegel proved a celebrated theorem on finiteness for integral solutions of certain diophantine equations. This theorem applies to systems of polynomial equations which either (a) describe an irreducible curve whose projective closure has positive genus, or (b) describe an irreducible curve of genus zero with at least three points at infinity. For such systems, Siegel's theorem says that there are only finitely many solutions in the ring of integers of any given number field.

Siegel's proof used the method of diophantine approximations, as pioneered by Thue in 1909 [T]. To give an example, if $(x, y)$ is a large integral solution of the equation $x^{3}-2 y^{3}=1$, then

$$
\left|\frac{x}{y}-\sqrt[3]{2}\right|=\frac{1}{|y|\left|x^{2}+\sqrt[3]{2} x y+\sqrt[3]{4} y^{2}\right|} \ll \frac{1}{|y|^{3}} .
$$

By continued fractions, for any irrational real number $\alpha$ there exists a constant $c=c(\alpha)$ and infinitely many rational numbers $x / y(x, y \in \mathbb{Z})$ with $|x / y-\alpha|<c /|y|^{2}$. Thue and Siegel used a weak converse of this fact to obtain their finiteness statements. Other mathematicians successively refined these estimates, until Roth proved in 1955 [R] that for any algebraic number $\alpha$ and any $c>0$ and $\varepsilon>0$, there exist only finitely many rational numbers $x / y$ $(x, y \in \mathbb{Z})$ with

$$
\left|\frac{x}{y}-\alpha\right|<\frac{c}{|y|^{2+\varepsilon}} .
$$

This did not lead to stronger diophantine statements, however. In fact, irreducible curves which are not of the type treated in Siegel's theorem are known to have infinitely many integral points over a sufficiently large ring.

On the other hand, one may also consider the question of rational points on irreducible curves. In this case, if the genus is at most one, then the curve has infinitely many rational points on a sufficiently large number field; if the genus is larger, then the curve has only finitely many rational points over any fixed number field. This latter assertion was conjectured by Mordell in 1922 and proved by Faltings in 1983 [F 1].

Faltings' proof was completely independent of diophantine approximation and Thue's method. From the points of view of both Nevanlinna theory and

Received by the editors May 28, 1991.

1991 Mathematics Subject Classification. Primary 11D75; Secondary 11J25, 14G40.

Partially supported by NSF grants DMS-8610730 and DMS-9001372. 
Arakelov intersection theory, however, the notions of integral points and rational points are very closely related. This suggests that one should also be able to prove Mordell's conjecture using Thue's method; this was accomplished by the present author recently [V 4]. However, that proof applied only to rational points on curves: it was still separate from any proof of Roth's theorem.

Part of the goal of the present paper, then, is to give a unified proof of both Roth's theorem and Mordell's conjecture.

The other part of the goal is a bit more technical. For algebraic points on a curve, one can define a measure of the complexity of these points, called the height. This height has the property that if all points in a given set are defined over a fixed number field, and if their heights are bounded, then the set is finite. Thus Mordell's conjecture can be phrased as the assertion that, given a curve of genus greater than one and a number field $k$, there exists a bound on the heights of $k$-rational points. Ideally, one would hope to strengthen this bound by showing that as $k$ varies, the bound varies linearly in the logarithm of the discriminant of $k$. This is a bit ambitious, though: as is shown in [V 1, 5.ABC], this would imply that the Fermat conjecture holds for all but finitely many exponents.

Instead, for an algebraic point $P$ on a curve, we use the arithmetic discriminant defined in [V 5]. This is greater than the normalized logarithm of the discriminant of $k(P)$. The difference corresponds to singularities in the closure of $P$ on an arithmetic surface; in the function field case this is merely the difference between the arithmetic and geometric genera of a curve. For details on this, see [V 5].

Thus the second goal of this paper is to prove a weaker version of the bound on heights of algebraic points, using the arithmetic discriminant of $P$ in place of the normalized log of the discriminant of $k(P)$. Again, this bound also holds for curves of genus zero; in this special case it reduces to a generalization of Roth's theorem, which was already proved by Wirsing [W].

We now make the above discussion more precise.

Let $C$ be a curve defined over a number field $k$, let $K$ be a canonical divisor on $C$, and let $\pi: X \rightarrow B$ be a regular model, where $B$ is the arithmetic curve corresponding to the ring of integers of $k$ (as in [V 4]). For each infinite place $\sigma \in B_{\infty}$ (i.e., $\sigma: k \hookrightarrow \mathbb{C}$ ), let $\mu_{\sigma}$ be the (Arakelov) canonical volume form on $C_{\sigma}:=C \times_{\sigma} \mathbb{C}$ for which the adjunction formula holds. For algebraic points $P \in C(\bar{k})$, let $H_{P}$ be the corresponding prime horizontal Arakelov divisor on $X$. Fix a finite set $S$ of places of $k$. For arithmetic divisors $D$ on $X$ let

$$
h_{D}(P)=\frac{\left(H_{P} \cdot D\right)}{[k(P): \mathbb{Q}]}
$$

and for $P \notin \operatorname{Supp} D$ let

$$
m(D, P)=\frac{1}{[k(P): \mathbb{Q}]} \sum_{v \in S}\left(H_{P} . D\right)_{v} .
$$

Let $\omega_{X / B}$ be the dualizing sheaf, with admissible metric. Then let

$$
d_{\mathrm{a}}(P)=\frac{\left(H_{P} \cdot H_{P}+\omega_{X / B}\right)}{[k(P): \mathbb{Q}]}
$$


and let $h_{K}(P)$ be the height relative to $\omega_{X / B}$ with this metric.

Then the main theorem of this paper is the following.

Theorem 0.1. In addition to the above notation, fix an integer $\nu \geq 1$, a real number $\varepsilon>0$, an effective divisor $D$ on $X$ with no multiple components, and a divisor $A$ on $X$ which is ample on the generic fibre. Then for all points $P \in C(\bar{k}) \backslash \operatorname{Supp} D$ with $[k(P): k] \leq \nu$,

$$
m(D, P)+h_{K}(P) \leq d_{\mathrm{a}}(P)+\varepsilon h_{A}(P)+O(1),
$$

where the constant in $O(1)$ depends on $X, D, \nu, A$, and $\varepsilon$.

This result was proved in a very weak form in [V 4, 7.1], and conjectured in nearly the above form (with $D=0$ ) in [V 5]. When $\nu=1$ this theorem gives a statement for rational points on $C$, which is equivalent to Roth's theorem when $g=0$, and Mordell's conjecture when $g>1$ and $D=0$. Thus, this paper gives a unified proof of Roth's theorem and Mordell's conjecture. Also, in the case $g=1$ it gives an approximation statement for rational points on elliptic curves; this is already known as an intermediate step in the derivation of Siegel's theorem from Roth's theorem.

When $g=0$ and $\nu$ is arbitrary, this result contains Wirsing's generalization [W] of Roth's theorem:

Corollary 0.2. Fix $\nu$ and $\varepsilon$ as above, and fix $\alpha \in \overline{\mathbb{Q}}$. Then there are at most finitely many $x \in \overline{\mathbb{Q}}$ with $[\mathbb{Q}(x): \mathbb{Q}] \leq \nu$ such that

$$
|x-\alpha|<H(x)^{-2 \nu-\varepsilon} \text {. }
$$

Working in another direction, as noted in [V 5], Theorem 0.1 with $D=0$ implies the following corollaries.

Corollary 0.3. Let $C$ be a curve of genus $g$ defined over a number field $k$, let $\nu$ be a positive integer, and let $f: C \rightarrow \mathbb{P}^{1}$ be a dominant morphism. Assume that

Then the set

$$
g-1>(\nu-1) \operatorname{deg} f .
$$

$$
\{P \in C(\bar{k}) \mid[k(P): k] \leq \nu \text { and } k(f(P))=k(P)\}
$$

is finite.

Corollary 0.4. If $C$ is a curve of genus $\geq 6$, then there exists a dominant morphism $f: C \rightarrow \mathbb{P}^{1}$ such that the set

$$
\{P \in C(\bar{k}) \mid[k(P): k] \leq 2 \text { and } k(f(P))=k(P)\}
$$

is finite.

Corollary 0.5. If $C$ is a hyperelliptic curve of genus $\geq 4$, then all but finitely many points on $C$ defined over a quadratic extension of $k$ are those obtained from $k$-rational points on $\mathbb{P}^{1}$ by pulling back via the canonical morphism $C \rightarrow \mathbb{P}^{1}$ of degree two.

Corollary 0.5 has already been proved by Faltings [F 2]. A weaker form of it (giving a growth result for the heights of quadratic points instead of a finiteness result, but with $g \geq 5$ ) had already been proved by Silverman [Sil]. 
Theorem 0.1 is also a partial solution of [V 1, Conjecture 5.2.6b], which in the case of curves asserts that

$$
m(D ; P)+h_{K}(P) \leq d(P)+\varepsilon h(P)+O(1),
$$

where

$$
d(P)=\frac{\log \left|D_{k(P)}\right|}{[k(P): \mathbb{Q}]} .
$$

To see how this implies the asymptotic Fermat conjecture, see [V 1, 5.ABC].

A result analogous to Theorem 0.1 holds over function fields, by a completely analogous proof. But, for clarity, the proof will be given only for number fields. Also, we note that [V 2] contains a result similar to Theorem 0.1 , where $(0.1 .1)$ is replaced by

$$
h_{K}(P) \leq(2+\varepsilon) d(P)+O(1) .
$$

This result is valid only in the function field case, but it eliminates the restriction on the degree $[k(P): k]$, and it gives the much stronger bound using $d(P)$.

The proof of Theorem 0.1 is by Thue's method, as refined by Siegel, Dyson, and Roth. To this we add ideas of Wirsing, to deal with algebraic points, and [V 3], to deal with curves of higher genus. The early steps in the proof also use ideas of Faltings [F 2] to prove ampleness of a certain line sheaf and to construct a certain section of that sheaf. Thus we avoid many of the technical points, such as bounds on cohomology on the local fibres, which appear in [V 4]. Later parts of the proof call on ideas of Bombieri [B]; in particular his use of Roth's lemma in the last parts of the proof appears here as well.

As is usually the case with this method, the bounds on the heights of points are ineffective: it is only possible to bound the number of exceptions to a given inequality of type $(0.1 .1)$.

\section{NOTATIONS AND CONVENTIONS}

Fields and absolute values. Throughout this paper (with the exception of $\S 2$ ), $k$ will be a fixed number field and $R$ its ring of integers. By a place of $k$, we mean either (i) an embedding $\sigma: k \hookrightarrow \mathbb{C}$ (an archimedean place), or (ii) a nonzero prime ideal $\mathfrak{p}$ of $R$ (a non-archimedean place). At these places we have corresponding absolute values: for $x \in k \backslash\{0\}$ and places $v$ of $k$, let

$$
|x|_{v}= \begin{cases}|\sigma(x)| & \text { if } v \text { corresponds to } \sigma: k \hookrightarrow \mathbb{C}, \text { or } \\ p^{-\left[k_{\mathfrak{p}}: \mathbb{Q}_{p}\right] \text { ord }_{\mathfrak{p}} x} & \text { if } v \text { corresponds to a prime ideal } \mathfrak{p} \\ & \text { containing the rational prime } p\end{cases}
$$

Note in particular that if $\sigma: k \hookrightarrow \mathbb{C}$ is a nonreal embedding, then $\sigma \neq \bar{\sigma}$, but the absolute values coincide. To make the formulas fit, we also set $k_{v}=\mathbb{C}$ for all archimedean places $v$ of all number fields $k$.

Arithmetic schemes. Many parts of this paper will refer to arithmetic schemes. These are defined in [V 4], but in the present case only the notions of metrized line sheaves and their corresponding arithmetic divisors, as well as the definitions of the schemes themselves, will be needed. 
Let $\mathscr{L}$ be a metrized line sheaf on an arithmetic scheme $X$. Unlike [V 4], which uses the $L^{2}$ norm of sections of $\mathscr{L}$, it is convenient here to use exclusively the sup norm, which is defined as

for all infinite places $\sigma$.

$$
\|s\|_{\text {sup }, \sigma}=\sup _{P \in X_{\sigma}}\|s(P)\|
$$

Let $B$ be the arithmetic scheme corresponding to $k$. The curve $C$ is assumed to be defined over $k$, so we may let $\pi: X \rightarrow B$ be the corresponding arithmetic surface, which is assumed to be regular.

For each archimedean place $\sigma$ of $k$ let $\mu_{\sigma}$ be the canonical volume form on $C_{\sigma}:=C \times_{\sigma} \mathbb{C}$. Horizontal prime divisors $H_{P}$ on $X$ corresponding to algebraic points $P \in C(\bar{k})$ will be defined as Arakelov divisors relative to these volume forms. Also let $\omega_{X / B}$ be the relative dualizing sheaf, with admissible metrics, and let the canonical divisor $K$ on $C$ be extended to $X$ via this metric, so that

$$
h_{K}(P)=\frac{\left(H_{P} \cdot \omega_{X / B}\right)}{[k(P): \mathbb{Q}]} .
$$

Arakelov theory also provides a Green's function $G_{\sigma}: C_{\sigma} \times C_{\sigma} \rightarrow \mathbb{R}_{\geq 0}$, normalized so that $G_{\sigma}(P, Q) /|z(P)-z(Q)|$ extends continuously along $P=Q$ if $z: C_{\sigma} \rightarrow \mathbb{C}$ is a local coordinate patch on $C_{\sigma}$. Also let $g_{\sigma}(P, Q)=$ $-\log G_{\sigma}(P, Q)$, so that $g_{\sigma}\left(P_{\sigma}, Q_{\sigma}\right)$ is the local intersection number $\left(H_{P}, H_{Q}\right)_{\sigma}$ if $P, Q \in C(k)$. Likewise, at non-archimedean places $v$ it will sometimes be convenient to let $g_{v}: C\left(\bar{k}_{v}\right) \times C\left(k_{v}\right) \rightarrow \mathbb{R}$ be the local intersection number, and $G_{v}=\exp \left(-g_{v}\right)$. Note the asymmetry: $g_{v}(P, Q)$ is defined for $P \in C\left(\bar{k}_{v}\right)$ and $Q \in C\left(k_{v}\right)$ by pulling back the canonical section of $\mathscr{O}(Q)$ to the base change to $k_{v}(P)$ of the local fibre of $X$ at $v$, and taking its degree along $P$.

The proof will often make use of $\mathbb{Q}$-divisors, i.e., divisors with rational coefficients. Notions of intersection theory and ampleness extend to this case in a straightforward manner. We also will refer to the corresponding fractional powers of invertible sheaves; these will likewise be treated formally unless one tensors by a high enough power to cancel the denominators. The same comments apply to sections of a fractional power of a line sheaf.

Conjugates. At each place $v$ of $k$ an algebraic point $P \in C(\bar{k})$ has $[k(P): k]$ conjugates in $C\left(\bar{k}_{v}\right)$; these will be denoted $P^{[\alpha]} \in C\left(\bar{k}_{v}\right)$ for $\alpha=1, \ldots$, $[k(P): k]$. Thus

$$
\left(H_{P} \cdot H_{Q}\right)_{v}=\sum_{\alpha} g_{v}\left(P^{[\alpha]}, Q_{v}\right) .
$$

(Of course the $P^{[\alpha]}$ will also depend on $v$, but in each case the choice of $v$ will be clear, so it will be omitted from the notation.) By a slight abuse of notation we will also write $g_{v}(-, Q)$ in place of $g_{v}\left(-, Q_{v}\right)$ for $Q \in C(k)$, and $g_{v}(-, D)$ in place of $\sum_{Q} n_{Q} g_{v}\left(-, Q_{v}\right)$ for divisors $D=\sum_{Q} n_{Q} \cdot Q$ on $X$ if Supp $D \subseteq C(k)$. Similar conventions will apply to $G_{v}$.

Other comments. When dealing with products $C^{n}$ or $X \times_{B} \cdots \times_{B} X$, subscripts will often be used to distinguish between the factors: $C_{i}, \pi_{i}, X_{i}$. Also, the notation $\mathrm{pr}_{i}$ will denote the projection onto the $i$ th factor. 
Several kinds of constants appear in the proof. First there are the $\varepsilon_{i}$, which as one might expect are taken small. Then we have constants $c_{i}$. They are often introduced as $c_{i}=c_{i}(a, b, c, \ldots)$, indicating that they depend on $a, b, c, \ldots$. These constants are often divided by the height of a point, especially when being compared with some $\varepsilon$ 's. Thus they operate at a lower level. At the lowest level are the constants $o(d)$ and $o(1)$, which depend on all data except $d$ (and $d_{1}, \ldots, d_{n}$ ); the indicated limiting behavior holds for $d$ sufficiently large and divisible. Often we will give explicit formulas for various constants, but the purpose in doing so is to possibly make the proof more understandable, rather than to carry out any explicit computations.

\section{REDUCTIONS AND CONSTRUCTIONS}

In this section we show that certain additional assumptions may be made in proving Theorem 0.1 , and we define some of the objects to be used in that proof.

Lemma 2.1. It will suffice to prove Theorem 0.1 under the additional assumptions

(i) all points in $\operatorname{Supp} D$ are defined over $k$;

(ii) the map $j: C \rightarrow J$ defined just prior to Lemma 6.5 is defined over $k$;

(iii) the curve $C$ has semistable reduction over $k$;

(iv) nodes on the singular fibres of $\pi: X \rightarrow B$ are defined over their respective residue fields, and the tangent directions of the fibre components passing through those nodes are also rational over the residue fields; and

(v) the divisor $K+D$ is ample on $C$.

Proof. Both sides of the inequality are invariant under base change (up to $O(1)$ ); therefore we may enlarge $k$ until the additional assumptions (i)-(iv) hold.

As for (v), if $K+D$ is not ample, then the theorem is trivial:

$$
m(D, P)+h_{K}(P) \leq h_{D}(P)+h_{K}(P)+O(1) \leq \varepsilon h_{A}(P)+O(1)
$$

by $[\mathrm{V} 1,1.2 .9 \mathrm{~d}]$. C.

By (iii), we assume from now on that $X$ is the regular semistable model for

Lemma 2.2 [W, Lemma 18]. If Theorem 0.1 is false, then the inequality fails to hold for an infinite set $\mathscr{P}$ of points such that $[k(P): k]=\nu$ for all $P \in \mathscr{P}$, and such that these fields are all linearly disjoint over $k$ (i.e., for any finite set $\left.\left\{P_{1}, \ldots, P_{n}\right\} \subseteq \mathscr{P},\left[k\left(P_{1}, \ldots, P_{n}\right): k\right]=\nu^{n}\right)$. (This reduction may involve enlarging $k$ or shrinking $\nu$.)

Now let $F$ be the $\mathbb{Q}$-divisor $K /(2 g-2)$, and for points $P \in C(\bar{k})$ let

$$
h(P):=h_{F}(P)=\frac{\left(H_{P} \cdot F\right)}{[k(P): \mathbb{Q}]}
$$

be the height of that point. Then

$$
h_{K}(P)=(2 g-2) h(P) \text {. }
$$


We also note that it will suffice to replace $h_{A}(P)$ with $h(P)$, and therefore to prove that the exceptions to the inequality

$$
m(D, P)+h_{K}(P) \leq d_{\mathrm{a}}(P)+\varepsilon h(P)
$$

have bounded height.

As noted in [V 4], the scheme $X \times_{B} X$ is not regular: its singularities are exactly those points lying over a node on the fibres on both copies of $X$. Let $x_{1}$ and $y_{1}$ (resp. $x_{2}$ and $y_{2}$ ) be functions in the completed local ring at such nodes which generate the branches of the fibre; then adjoining the functions $x_{1} / x_{2}$ and $x_{2} / x_{1}$ on different open affine subsets gives a scheme $W_{2}$ which is regular and birational to $X \times_{B} X$. The following lemma gives a similar desingularization for $X^{n}:=X \times_{B} \cdots \times_{B} X$.

To keep the notation relatively simple, we assume that the choices of the functions $x_{i}$ and $y_{i}$ are chosen compatibly, independent of $i$.

Lemma 2.4. Let $\sigma$ be a permutation of $\{1, \ldots, n\}$. Let $f_{\sigma}: W_{2} \times_{B} X^{n-2} \rightarrow X^{n}$ be the birational morphism obtained by composing the permutation $\sigma$ applied to the factors of $X^{n}$ with the natural map $W_{2} \times{ }_{B} X^{n-2} \rightarrow X^{n}$. Let $W$ be the scheme which is the product over all permutations $\sigma$ of the schemes $W_{2} \times_{B} X^{n-2}$ via the maps $f_{\sigma}$. Then $W$ is a regular scheme.

In other words, $W$ is the smallest scheme which maps morphically onto $W_{2}$ by choosing any distinct pair of factors.

Proof. Fix $P \in W$. To show that $W$ is regular at $P$, we may assume that $P$ lies over a node on the fibres of all factors $X$. Otherwise, since $W_{2}$ is isomorphic to $X \times{ }_{B} X$ except for above nodes on both factors, $W$ is locally isomorphic to the product of $X$ with the scheme $W_{n-1}$ obtained by desingularizing $X^{n-1}$, and we are done by induction.

For $i=1, \ldots, n$ let $x_{i}$ and $y_{i}$ be the locally defining functions for the local branches of the fibre on each factor $X_{i}$, at the node underlying $P$. We may assume that $x_{i} y_{i}=t$ for some fixed uniformizer $t$ on the completed local ring $\mathscr{O}_{B, v}$ of $B$. By construction of $W_{2}$, for each pair $i<j$ of indices, at least one of the functions $x_{i} / x_{j}$ or $x_{j} / x_{i}$ is regular at $P$. Let that function be denoted $w_{i j}$. Let $R \subseteq\{1, \ldots, n\}^{2}$ be the relation given by $(i, j) \in R$ if $w_{i j}=x_{i} / x_{j}$; otherwise $(j, i) \in R$. If $R$ does not satisfy the axioms for an order, then there exists some set $i_{1}, \ldots, i_{r}$ such that $\left(i_{1}, i_{2}\right), \ldots,\left(i_{n-1}, i_{n}\right),\left(i_{n}, i_{1}\right)$ all lie in $R$, which implies that the product of the corresponding $w$ 's is 1 . We may then replace some $w$ with its reciprocal and repeat until $R$ becomes an ordering.

Then $R$ is associated with some permutation $\sigma$ : for all $i$ and $j,(\sigma(i), \sigma(j))$ $\in R$ if and only if $i<j$. The completed local ring $\mathscr{O}_{W, P}$ is therefore the quotient of the ring

$$
\mathscr{O}_{B, v}\left[\left[y_{\sigma(1)}, w_{\sigma(1), \sigma(2)}, \ldots, w_{\sigma(n-1), \sigma(n)}, x_{\sigma(n)}\right]\right]
$$

by the ideal

$$
\left(y_{\sigma(1)} w_{\sigma(1), \sigma(2)} \cdots w_{\sigma(n-1), \sigma(n)} x_{\sigma(n)}-t\right) .
$$

Thus it is regular. 
For $i=1, \ldots, n$ let $\mathrm{pr}_{i}: W \rightarrow X_{i}$ be the morphism corresponding to the projection $\mathrm{pr}_{i}: C^{n} \rightarrow C_{i}$. For distinct indices $i$ and $\ell$, let $\mathrm{pr}_{i \ell}: W \rightarrow W_{2}$ be the map corresponding to $\operatorname{pr}_{i} \times \mathrm{pr}_{\ell}: W \rightarrow X_{i} \times{ }_{B} X_{\ell}$; it is a morphism by the definition of $W$.

The divisor $F$ has already been defined; also let $F_{i}=\operatorname{pr}_{i}^{*} F$. The diagonal $\Delta$ on $C \times C$ extends to $W_{2}$ by taking its closure; this gives a Cartier divisor since $W_{2}$ is regular. Then we let

$$
\Delta^{\prime}=\Delta-F_{1}-F_{2},
$$

where the divisors $F_{1}$ and $F_{2}$ on $W_{2}$ are defined analogously to those on $W$. Also let

$$
\Delta_{i \ell}=\operatorname{pr}_{i \ell}^{*} \Delta \text { and } \Delta_{i \ell}^{\prime}=\operatorname{pr}_{i \ell}^{*} \Delta^{\prime}
$$

for all $i \neq \ell$, so that $\Delta_{i \ell}^{\prime}=\Delta_{i \ell}-F_{i}-F_{\ell}$.

\section{Certain Polynomials}

Lemma 3.1. Fix $g \in \mathbb{Z}, g \geq 0$, and for $n \geq 1$ let the polynomials $U_{n}(X)$ and $V_{n}(X)$ be defined by

$$
U_{1}(X)=X, \quad V_{1}(X)=0
$$

and

$$
\left(\begin{array}{c}
U_{n+1} \\
V_{n+1}
\end{array}\right)=\left(\begin{array}{cc}
X & X \\
-\frac{g n}{X} & n
\end{array}\right)\left(\begin{array}{l}
U_{n} \\
V_{n}
\end{array}\right)
$$

Let $f_{n}(X)=U_{n}(X)+V_{n}(X)$. Then

$$
f_{n}(X)=\sum_{i=0}^{g}(-1)^{i}\left(\begin{array}{l}
g \\
i
\end{array}\right) \frac{n !}{(n-i) !}(X+g)^{n-i},
$$

using the convention that all terms involving factorials of negative integers in denominators are zero.

Proof. It will suffice to show that the sequences

$$
U_{n}(X)=X \sum_{i=0}^{g}(-1)^{i}\left(\begin{array}{l}
g \\
i
\end{array}\right)(X+g)^{n-i-1} \frac{(n-1) !}{(n-i-1) !}
$$

and

$$
V_{n}(X)=\sum_{i=0}^{g}(-1)^{i}\left(\begin{array}{l}
g \\
i
\end{array}\right)(X+g)^{n-i-1}\left((X+g) \frac{n !}{(n-i) !}-X \cdot \frac{(n-1) !}{(n-i-1) !}\right)
$$

satisfy (3.1.1) and (3.1.2). It is easy to check directly that these expressions satisfy (3.1.1), as well as the equation $U_{n+1}=X\left(U_{n}+V_{n}\right)$ from (3.1.2). It remains to check that they satisfy $V_{n+1}=-\frac{g n}{X} U_{n}+n V_{n}$. Evaluating the right- 
hand side gives

$$
\begin{aligned}
-\frac{g n}{X} U_{n}+n V_{n}= & n \sum_{i}(-1)^{i}\left(\begin{array}{l}
g \\
i
\end{array}\right)(X+g)^{n-i-1} \\
& \times\left(-g \cdot \frac{(n-1) !}{(n-i-1) !}+(X+g) \frac{n !}{(n-i) !}-X \cdot \frac{(n-1) !}{(n-i-1) !}\right) \\
= & \sum_{i}(-1)^{i}\left(\begin{array}{l}
g \\
i
\end{array}\right)(X+g)^{n-i}\left(n \cdot \frac{n !}{(n-i) !}-\frac{n !}{(n-i-1) !}\right) \\
= & \sum_{i}(-1)^{i}\left(\begin{array}{l}
g \\
i
\end{array}\right)(X+g)^{n-i}\left(i \cdot \frac{n !}{(n-i) !}\right) .
\end{aligned}
$$

By the above convention regarding factorials of negative integers, the sum can be taken over all $i \in \mathbb{Z}$. Thus

$$
\begin{aligned}
& V_{n+1}+\frac{g n}{X} U_{n}-n V_{n} \\
& =\sum_{i}(-1)^{i}\left(\begin{array}{c}
g \\
i
\end{array}\right)(X+g)^{n-i} \frac{n !}{(n-i+1) !}((X+g)(n+1)-(X+i)(n-i+1)) \\
& =\sum_{i}(-1)^{i}\left(\begin{array}{c}
g \\
i
\end{array}\right)(X+g)^{n-i} \frac{n !}{(n-i+1) !}((g-i)(n-i+1)+(X+g) \cdot i) \\
& =\sum_{i}(-1)^{i}\left((g-i)\left(\begin{array}{c}
g \\
i
\end{array}\right)(X+g)^{n-i} \frac{n !}{(n-i) !}\right. \\
& \left.\quad+(g-i+1)\left(\begin{array}{c}
g \\
i-1
\end{array}\right)(X+g)^{n-i+1} \frac{n !}{(n-i+1) !}\right)
\end{aligned}
$$

$=0$.

Remark. From the expression (3.1.3) it follows immediately that the polynomials $f_{n}$ are monic of degree $n$, and that $f_{n}^{\prime}=n f_{n-1}$.

Proposition 3.2. Fix an integer $g \geq 0$. For $n \geq 1$ let $\xi_{n}$ be the largest real root of $f_{n}(X)$. Then

(a) $\xi_{n}$ exists;

(b) $\xi_{n+1} \geq \xi_{n} \geq 0$;

(c) $\xi_{n} \leq n+o(n)$ as $n \rightarrow \infty$.

(d) Given $\varepsilon_{1}>0$ there exists a constant $\varepsilon_{2}>0$ such that for all sufficiently large $n$,

$$
\xi_{n}<(n-1)\left(1+\varepsilon_{1}\right)
$$

and

$$
f_{n}\left((n-1)\left(1+\varepsilon_{1}\right)\right)>\varepsilon_{2}\left((n-1)\left(1+\varepsilon_{1}\right)\right)^{n} .
$$

Proof. First, note that

$$
f_{n+1}(X)=(X+n) f_{n}(X)-n(X+g) f_{n-1}(X) .
$$

Indeed, this follows from (3.1.3) by an argument similar to the end of the proof of Lemma 3.1. The details are left to the reader. 
Now, to prove (a) and (b), it will suffice to show that $f_{n+1}\left(\xi_{n}\right) \leq 0$. By (3.2.2), it suffices to show that $f_{n-1}\left(\xi_{n}\right) \geq 0$, which follows by induction on $n$.

To show (c), first note that if $g=0$ then $f_{n}(X)=X^{n}$, so that $\xi_{n}=0$ for all $n$. If $g>0$, then write

$$
\begin{aligned}
j_{g, n}(y) & =\frac{1}{n^{g}} \sum_{i}(-1)^{i}\left(\begin{array}{l}
g \\
i
\end{array}\right) \frac{n !}{(n-i) !}(n(1+y))^{g-i} \\
& =\sum_{i}(-1)^{i}\left(\begin{array}{c}
g \\
i
\end{array}\right)\left(\frac{n}{n}\right)\left(\frac{n-1}{n}\right) \cdots\left(\frac{n-i+1}{n}\right)(1+y)^{g-i},
\end{aligned}
$$

so that

$$
f_{n}(X)=n^{g}(X+g)^{n-g} j_{g, n}\left(\frac{X+g}{n}-1\right) .
$$

Then it will suffice to show that the largest real root $y_{g, n}$ of $j_{g, n}$ satisfies

$$
y_{g, n} \leq o_{g}(1)
$$

as $n \rightarrow \infty$. This is proved by induction on $g$, the case $g=1$ being immediate. For $g>1$, it is easy to check that

$$
j_{g, n}^{\prime}(y)=g j_{g-1, n}(y) \text {. }
$$

Therefore it suffices to show that for any $\varepsilon>0$ and any $n$ sufficiently large (depending on $g$ and $\varepsilon$ ), we have $j_{g, n}(\varepsilon)>0$. This holds because

$$
\lim _{n \rightarrow \infty} j_{g, n}(\varepsilon)=\sum_{i=0}^{g}(-1)^{i}\left(\begin{array}{c}
g \\
i
\end{array}\right)(1+\varepsilon)^{g-i}=\varepsilon^{g}>0 .
$$

Finally, to prove (d), note that rewriting (3.2.1) in terms of $j_{g, n}$ gives the condition

$$
j_{g, n}\left(\varepsilon_{1}+\frac{g-1-\varepsilon_{1}}{n}\right)>\varepsilon_{2}\left(\frac{(n-1)\left(1+\varepsilon_{1}\right)}{n}\right)^{g}\left(\frac{(n-1)\left(1+\varepsilon_{1}\right)}{(n-1)\left(1+\varepsilon_{1}\right)+g}\right)^{n-g} .
$$

Then, by (3.2.3), it will suffice to take $\varepsilon_{2}<\left(\varepsilon_{1} /\left(1+\varepsilon_{1}\right)\right)^{g}$.

\section{AMPLENESS OF A DIVISOR}

We will be working with tuples $(h)$ satisfying the following condition.

Condition 4.1. The tuple $(h)=\left(h_{1}, \ldots, h_{n}\right)$ is composed of positive numbers for which $\sqrt{h_{i} h_{\ell}} \in \mathbb{Q}$ for all $i, \ell$.

For such tuples, and for rational $x$, let

$$
Y_{x,(h)}=x \sum_{i=1}^{n} \frac{F_{i}}{h_{i}}+\sum_{i<\ell} \frac{\Delta_{i \ell}^{\prime}}{\sqrt{h_{i} h_{\ell}}} .
$$

Proposition 4.3. We have

$$
Y_{x,(h)}^{n}=\frac{n ! f_{n}(x)}{h_{1} h_{2} \cdots h_{n}}
$$


where $f_{n}$ is the polynomial defined in Lemma 3.1.

The following two lemmas contain all the intersection theory needed to evaluate $Y_{x,(h)}^{n}$. Note that $\Delta_{i \ell}^{\prime 2}=-2 g F_{i} F_{\ell}$ and $F_{i}^{2}=\Delta_{i \ell}^{\prime} . F_{i}=\Delta_{i \ell}^{\prime} . F_{\ell}=0$.

Lemma 4.3.1. Any product of $n$ factors, each of the form $F_{i}$ or $\Delta_{i \ell}^{\prime}$, is zero unless each index occurs either exactly once as a subscript of $F$, or exactly twice as a subscript of $\Delta^{\prime}$.

Proof. Assume that the product is nonzero. Since $F_{i}^{2}=F_{i} \cdot \Delta_{i \ell}^{\prime}=0$, indices appearing as subscripts of $F$ may not occur as subscripts of $\Delta^{\prime}$, and may occur only once. But, each index $i$ must occur at least once in the product; otherwise the product is the pull-back of a cycle of codimension $n$ from $C_{1} \times \cdots \times \widehat{C}_{i} \times$ $\cdots \times C_{n}$, and hence it is zero. To conclude the proof of the lemma, it remains only to rule out the case that some index $i$ occurs only once as a subscript of some $\Delta_{i \ell}^{\prime}$. In that case the intersection number of the product is the intersection of some $\Delta_{i \ell}^{\prime}$ with a cycle coming from $C_{1} \times \cdots \times \widehat{C}_{i} \times \cdots \times C_{n}$, and again it is zero.

Lemma 4.3.2. For any distinct indices $i_{1}, \ldots, i_{r}(r \geq 2)$, we have

$$
\Delta_{i_{1} i_{2}}^{\prime} \cdots \Delta_{i_{r-1} i_{r}}^{\prime} \Delta_{i_{r} i_{1}^{\prime}}=-2 g
$$

on $C_{i_{1}} \times \cdots \times C_{i_{r}}$.

Proof. This will be proved by induction on $r$, the case $r=2$ being easily checked. For $r>2$, we may assume that $i_{1}=1, i_{2}=2$, etc. Now

$$
\Delta_{12}^{\prime} \cdot \Delta_{23}^{\prime}\left(\Delta_{34}^{\prime} \cdots \Delta_{r 1}^{\prime}\right)=\operatorname{pr}_{\hat{2} *}\left(\Delta_{12}^{\prime} \cdot \Delta_{23}^{\prime}\right) \cdot\left(\Delta_{34}^{\prime} \cdots \Delta_{r 1}^{\prime}\right)
$$

where $\mathrm{pr}_{\hat{2}}$ denotes the projection onto $C_{1} \times C_{3} \times \cdots \times C_{r}$ and the last factor on the right is interpreted as a cycle on $C_{1} \times C_{3} \times \cdots \times C_{r}$. But

$$
\operatorname{pr}_{\hat{2} *}\left(\Delta_{12}^{\prime} \cdot \Delta_{23}^{\prime}\right)=\operatorname{pr}_{\hat{2} *}\left(\left(\Delta_{12}-F_{1}\right)\left(\Delta_{23}-F_{3}\right)\right)=\Delta_{13}-F_{1}-F_{3}=\Delta_{13}^{\prime}
$$

since $F_{2} \cdot \Delta_{23}^{\prime}=F_{2} \cdot\left(\Delta_{12}-F_{1}\right)=0$ and $\operatorname{pr}_{\hat{2} *}\left(F_{1} \cdot F_{3}\right)=0$. Thus the expression is reduced to $\Delta_{13}^{\prime} \Delta_{34}^{\prime} \cdots \Delta_{r 1}^{\prime}$, which equals $-2 g$ by induction.

Proof of Proposition 4.3. By the first lemma, $Y_{x,(h)}^{n}$ is homogeneous of degree -1 in each $h_{i}$, so we may assume that all $h_{i}=1$. Thus it will suffice to show, by induction on $n$, that $Y_{x,(h)}^{n}=n ! f_{n}(x)$. For $n \geq 1$, let $n ! U_{n}(x)$ be the sum of all terms in the expansion of $Y_{x,(h)}^{n}$ containing a factor $F_{n}$; let $n ! V_{n}(x)$ be the sum of all remaining terms. It is easy to check that $U_{1}(x)=x$ and $V_{1}(x)=0$. It remains only to check that $U_{n+1}$ and $V_{n+1}$ satisfy (3.1.2).

To do this, imagine that the expression for $Y_{x,(h)}^{n+1}$ on $C^{n+1}$ is multiplied out and all terms which evaluate to zero by the first lemma are crossed out, but that the commutative law of intersection products is not used. Collecting those terms involving $F_{n+1}$, we see that

$$
(n+1) ! U_{n+1}(x)=x(n+1) \cdot n ! f_{n}(x),
$$


as desired. (The factor $n+1$ comes from the fact that $x F_{n+1}$ can be removed from $n+1$ different places in a term, giving the same term in $n ! f_{n}(x)$.) Likewise,

$$
(n+1) ! V_{n+1}(x)=-\frac{g n(n+1)}{x} \cdot n ! U_{n}(x)+n(n+1) \cdot n ! V_{n}(x) .
$$

The first term comes from replacing $\Delta_{i, n+1}^{\prime 2}$ with $F_{i}$ and interchanging the indices $i$ and $n$; the second term comes from replacing $\Delta_{i, n+1}^{\prime} \Delta_{\ell, n+1}^{\prime}$ with $\Delta_{i \ell}^{\prime}$ and interchanging $i$ and $n$. Thus, the proposition follows.

Theorem 4.4. Fix $n \geq 1$ and $g \geq 0$, and assume that $x>\xi_{n}$. Then there exists a number $r>1$ (depending on $g, n$, and $x$ ) such that if

$$
h_{i+1} / h_{i}>r \quad \text { for all } i=1, \ldots, n,
$$

then $Y_{x,(h)}$ is ample.

Proof. The proof is similar to the proof of [F 2, Theorem 4.4]. It proceeds by induction on $n$, using the fact that $x>\xi_{n-1}$. As in the proof of Faltings, we find that for $d$ sufficiently large and divisible, $\Gamma\left(C^{n}, \mathscr{O}\left(d Y_{x,(h)}\right)\right) \neq 0$. As in [V 3, 1.5], we find that if $\delta>0$ and $s \gg 0$, then

$$
-\frac{\Delta_{i \ell}^{\prime}}{\sqrt{h_{i} h_{\ell}}}+\frac{\sqrt{g+\delta} F_{i}}{h_{i}}+\frac{\sqrt{g+\delta} F_{\ell}}{h_{\ell}}
$$

is ample on $C_{i} \times C_{\ell}$, hence is generated by its global sections on $C^{n}$. It follows that there are injections

$$
\mathscr{O}\left(d Y_{x,(h)}\right) \hookrightarrow \mathscr{O}\left(\sum e_{i} F_{i}\right)
$$

without common zero, where

$$
e_{i}=\frac{d(x+(n-1) \sqrt{g+\delta})}{h_{i}} .
$$

The rest of the proof proceeds as in [F 2].

\section{Dimension COUNTING}

In Bombieri's argument ampleness per se is not important; it is only necessary to find a lower bound on the dimension of the space of global sections. From the results of the previous two sections we know $h^{0}\left(C^{n}, d Y_{x,(h)}\right)$ for large $d$, but the desired space of sections will be reduced still further. We shall obtain a bound on this subspace of $H^{0}\left(C^{n}, d Y_{x,(h)}\right)$ by means of a lemma due to Roth and Wirsing.

Definition 5.1. Let $\xi$ be a point on $C^{n}$. For $i=1, \ldots, n$ let $x_{i}$ be a local coordinate on $C$ near $\operatorname{pr}_{i}(\xi)$ with $x_{i}\left(\operatorname{pr}_{i}(\xi)\right)=0$. By abuse of notation think 
of the $x_{i}$ as local coordinates on $C^{n}$ at $\xi$. Let $s$ be a section of a line sheaf $\mathscr{L}$ defined in a neighborhood of $\xi$ by a formal power series

$$
P\left(x_{1}, \ldots, x_{n}\right)=\sum_{\ell_{1}, \cdots, \ell_{n} \geq 0} a_{\ell_{1} \cdots \ell_{n}} x_{1}^{\ell_{1}} \cdots x_{n}^{\ell_{n}} .
$$

Let $d_{1}, \ldots, d_{n}$ be positive integers. Then the index of $s$ at $\xi$ relative to $d_{1}, \ldots, d_{n}$ is the real number

$$
t\left(s, \xi, d_{1}, \ldots, d_{n}\right):=\min \left\{\frac{\ell_{1}}{d_{1}}+\cdots+\frac{\ell_{n}}{d_{n}} \mid a_{(\ell)} \neq 0\right\} .
$$

If $Y$ is an effective divisor on $C^{n}$, then let its index be defined by means of a global section $s$ of $\mathscr{L}:=\mathscr{O}(Y)$ for which $Y=(s)$.

This definition is independent of the choice of local parameters.

Actually, we will not use this definition until much later in the paper; however, we introduce it here in order to motivate the following more technical definition.

Definition 5.2. Let $\xi, x_{1}, \ldots, x_{n}, d_{1}, \ldots, d_{n}, s$, and $a_{(\ell)}$ be as defined above. Also let $\phi:\{1, \ldots, n\} \rightarrow \mathbb{R}$ be a function. Then we say that $s$ has index function $\phi$ if for all $(\ell) \in \mathbb{Z}^{n}$ for which $a_{(\ell)} \neq 0$ and all $I \subseteq\{1, \ldots, n\}$, we have

$$
\sum_{i \in I} \frac{\ell_{i}}{d_{i}} \geq \phi(\# I)
$$

Also, we say that $s$ has truncated index function $\phi$ if (5.2.1) holds for all tuples $(\ell) \in \prod_{i=1}^{n}\left\{0, \ldots, d_{i}\right\}$ for which $a_{(\ell)} \neq 0$ and for all $I$.

In other words this gives a lower bound for the index, using any subset of the factors $C_{1}, \ldots, C_{n}$. Clearly the condition of having truncated index function $\phi$ is weaker than having (nontruncated) index function. Under certain conditions the converse also holds.

Lemma 5.3. Let $\xi, d_{1}, \ldots, d_{n}, s, \mathscr{L}$, and $a_{(\ell)}$ be as defined above. Assume also that $\left(C_{i} \cdot \mathscr{L}\right) \leq d_{i}$ for all $i=1, \ldots, n$. Let $(\ell)$ be the index of a leading term for $s$; i.e., $a_{(j)}=0$ for all $(j) \neq(\ell)$ with $j_{i} \leq \ell_{i}$ for all $i$. Then $\ell_{i} \leq d_{i}$ for all $i$. In particular, if $s$ has a given truncated index function $\phi$ relative to $d_{1}, \ldots, d_{n}$, then it also has (nontruncated) index function $\phi$.

Proof. The second assertion follows immediately from the first. To prove the first assertion, assume it is false for some tuple $(\ell)$. Without loss of generality, we may assume that $\ell_{1}>d_{1}$. Also, we may assume that $a_{(j)}=0$ for all $(j) \neq(\ell)$ satisfying $j_{i} \leq \ell_{i}$ for $i=2, \ldots, n$; otherwise we may decrease $\left(\ell_{2}, \ldots, \ell_{n}\right)$ and increase $\ell_{1}$.

Regard ${ }^{n} C_{2}, \ldots, C_{n}$ as a family over $C_{1}$, and let $\sigma$ be the $\left(\ell_{2}, \ldots, \ell_{n}\right)$ derivative of $s$ at $\left(\xi_{2}, \ldots, \xi_{n}\right)$, as defined in $\S 6$, below. Then $\sigma$ is a section of

$$
\left.\left(\mathscr{L} \otimes \operatorname{pr}_{2}^{*} \mathscr{O}\left(-\ell_{2} \xi_{2}\right) \otimes \cdots \otimes \operatorname{pr}_{n}^{*} \mathscr{O}\left(-\ell_{n} \xi_{n}\right)\right)\right|_{C_{1} \times \xi_{2} \times \cdots \times \xi_{n}}
$$

By the assumptions on $(\ell)$, this section is a nonzero section of a line sheaf of degree $d_{1}$ which has a zero of order $\ell_{1}>d_{1}$ at $\xi_{1}$. This is a contradiction. 
It follows from this lemma that a finite number of linear conditions are sufficient to imply the condition of having a certain index function. The following lemma gives a more precise estimate on the number of these linear conditions, for a certain function $\phi$.

Lemma 5.4 [W, Lemma 11]. Let $0<\varepsilon_{3}<1$, let $n>12 / \varepsilon_{3}^{4}$, and let $d_{1}, \ldots, d_{n}$ be integers with

$$
d_{i}>3 / \varepsilon_{3} \quad \text { for } i=1, \ldots, n .
$$

Then the number of elements in the set

$$
\begin{aligned}
& \left\{(l) \in \mathbb{Z}^{n} \mid 0 \leq \ell_{i} \leq d_{i} \text { for } i=1, \ldots, n\right. \\
& \left.\quad \text { and } \#\left\{i \mid \ell_{i} / d_{i} \leq x\right\} \geq n\left(x+\varepsilon_{3}\right) \text { for some real } x \in[0,1]\right\}
\end{aligned}
$$

is at most

$$
\varepsilon_{3} \prod_{i=1}^{n}\left(d_{i}+1\right)
$$

Corollary 5.5. For $\varepsilon_{3}, n$, and $d_{1}, \ldots, d_{n}$ as above, the number of elements in the set

$$
\begin{aligned}
& \left\{(l) \in \mathbb{Z}^{n} \mid 0 \leq \ell_{i} \leq d_{i} \text { for } i=1, \ldots, n\right. \\
& \left.\quad \text { and } \sum_{i \in I} \frac{\ell_{i}}{d_{i}}<\# I\left(\frac{\# I+1}{2 n}-\varepsilon_{3}\right) \text { for some } I \subseteq\{1, \ldots, n\}\right\}
\end{aligned}
$$

is at most

$$
\varepsilon_{3} \prod_{i=1}^{n}\left(d_{i}+1\right)
$$

Proof. It will suffice to show that the set of Lemma 5.4 contains the set of the corollary. Suppose $(\ell) \in \mathbb{Z}^{n}$ and $0 \leq \ell_{i} \leq d_{i}$ for $i=1, \ldots, n$, but that $(\ell)$ is not in the set of the lemma. Then

$$
\#\left\{i \mid \ell_{i} / d_{i} \leq x\right\}<n\left(x+\varepsilon_{3}\right) \quad \text { for all real } x \in[0,1] .
$$

Let $\eta_{1}, \ldots, \eta_{n}$ be a permutation of the $\ell_{i} / d_{i}, i=1, \ldots, n$, such that $\eta_{1} \leq$ $\cdots \leq \eta_{n}$. Then

$$
\eta_{i}>\frac{i}{n}-\varepsilon_{3}
$$

Indeed, let $x=i / n-\varepsilon_{3}$ in (5.5.1) (cf. [W, Lemma 12]). Then for all $I \subseteq$ $\{1, \ldots, n\}$,

$$
\sum_{i \in I} \frac{\ell_{i}}{d_{i}} \geq \sum_{i=1}^{\# I} \eta_{i} \geq \sum_{i=1}^{\# I}\left(\frac{i}{n}-\varepsilon_{3}\right) \geq \# I\left(\frac{\# I+1}{2 n}-\varepsilon_{3}\right) .
$$

We are now ready to prove the main result of this section. 
Proposition 5.6. Let $\varepsilon_{1}>0$ be given. Then there exists an integer $n_{0}>0$ and real constants $r>0, \varepsilon_{3}>0$, all depending on $\varepsilon_{1}$, with the following properties. For any $n \geq n_{0}$ let $x=(n-1)\left(1+\varepsilon_{1}\right)$. For all tuples $(h)$ satisfying $(4.1)$ and (4.4.1) and all sufficiently large and divisible $d=d\left(\varepsilon_{1}, n,(h)\right)$,

(a) we have

$$
h^{0}\left(C^{n}, d Y_{x,(h)}\right)>\varepsilon_{2} \cdot \frac{d^{n} x^{n}}{h_{1} \cdots h_{n}} ;
$$

(b) requiring that elements of this subspace have index function $\# I\left(\frac{\# I+1}{2 n}-\varepsilon_{3}\right)$ relative to $d_{i}:=d \cdot x / h_{i}$ at $(Q, Q, \ldots, Q)$ for all $Q \in \operatorname{Supp} D$ imposes at most

$$
\frac{\varepsilon_{2}}{2} \cdot \frac{d^{n} x^{n}}{h_{1} \cdots h_{n}}+O\left(d^{n-1}\right)
$$

linear conditions.

One would be tempted to combine the parts, saying that the subspace having the given index function as above has dimension at least $\left(\varepsilon_{2} / 2\right) \cdot\left(d^{n} x^{n} / h_{1} \cdots h_{n}\right)$. But this cannot be done until a later step, because we will actually be working with a subspace of $\Gamma\left(C^{n}, d Y_{x,(h)}\right)$.

Proof. By Proposition 3.2, there exists $\varepsilon_{2}>0$ such that for all sufficiently large $n$ we have $\xi_{n}<(n-1)\left(1+\varepsilon_{1}\right)$ and (3.2.1) holds. Let $n_{0}$ be an integer such that these conditions hold for all $n>n_{0}$; assume also that

$$
n_{0}>\frac{12}{\varepsilon_{3}^{4}} \quad \text { where } \quad \varepsilon_{3}:=\frac{\varepsilon_{2}}{2 \operatorname{deg} D}
$$

Then, for any $n \geq n_{0}$ let $x=(n-1)\left(1+\varepsilon_{1}\right)$. By Theorem 4.4 there exists a constant $r>0$ such that for all tuples $(h)$ satisfying (4.1) and (4.4.1), $Y_{x,(h)}$ is ample. It follows that for sufficiently large and divisible $d$,

$$
\begin{aligned}
h^{0}\left(C^{n}, d Y_{x,(h)}\right) & =\frac{d^{n}\left(Y_{x,(h)}^{n}\right)}{n !}+O\left(d^{n-1}\right) \\
& =\frac{d^{n} f_{n}(x)}{h_{1} \cdots h_{n}}+O\left(d^{n-1}\right) \\
& >\varepsilon_{2} \cdot \frac{d^{n} x^{n}}{h_{1} \ldots h_{n}}+O\left(d^{n-1}\right)
\end{aligned}
$$

by Proposition 4.3 and (3.2.1), respectively. Assuming that $d_{i}:=d \cdot x / h_{i}$ satisfy (5.4.1), it follows that imposing the extra "index function" condition reduces the dimension of the vector space by at most

$$
\operatorname{deg} D \cdot \varepsilon_{3} \cdot \prod_{i=1}^{n}\left(d_{i}+1\right)=\frac{\varepsilon_{2}}{2} \cdot \frac{d^{n} x^{n}}{h_{1} \cdots h_{n}} \prod_{i=1}^{n}\left(1+\frac{1}{d_{i}}\right) .
$$

This gives the bound (5.6.1). 


\section{Construction of a Global SECtion}

As is the case in the proofs of Bombieri and Faltings, we use Siegel's lemma to construct the desired section of $\mathscr{O}\left(d Y_{x, h)}\right)$. This involves considering the desired vanishing and compatibility conditions as a system of linear equations in the coefficients of the polynomials, and using Siegel's lemma to obtain a solution of small height. Actually, variants of Siegel's lemma due to both BombieriVaaler and Faltings are used here.

This argument replaces the use of the Gillet-Soule Riemann-Roch theorem in [V 4]. I do not know if it is possible to replace this argument with a use of Gillet-Soule: when dealing with a product of more than two curves it is difficult to control the cohomology over the local fibres of the number ring.

\section{Metrized modules.}

\section{Definition 6.1.}

(a) For vectors $\mathbf{x}=\left(x_{1}, \ldots, x_{\mathscr{N}}\right)$, define the height

$$
h(\mathbf{x})=\frac{1}{[k: \mathbb{Q}]} \sum_{v} \log \max _{i}\left|x_{i}\right|_{v} .
$$

(b) For an $\mathscr{M} \times \mathscr{N}$ matrix $A=\left(a_{i j}\right)$ in $k$ of rank $\mathscr{M}$, define the height of $A$ as the height of the vector consisting of the determinants of all $\mathscr{M} \times \mathscr{M}$ submatrices of $A$.

Lemma 6.2 (Siegel-Bombieri-Vaaler, [B-V, Theorem 9]). Let $A$ be an $\mathscr{M} \times \mathscr{N}$ matrix of rank $\mathscr{M}$ with entries in $k$. Then there exists a basis $\left\{\mathbf{x}_{1}, \ldots, \mathbf{x}_{\mathscr{N}-\mathscr{M}}\right\}$ of the kernel of $A$ (regarded as a linear transformation from $k^{\mathscr{N}}$ to $k^{\mathscr{M}}$ ) such that

$$
\sum_{i=1}^{\mathscr{N}-\mathscr{M}} h\left(\mathbf{x}_{i}\right) \leq h(A)+\frac{\mathscr{N}-\mathscr{M}}{[k: \mathbb{Q}]} \log \left(\left(\frac{2}{\pi}\right)^{s} \sqrt{\left|\Delta_{k}\right|}\right) .
$$

Here $s$ is the number of complex places of $k$, and $\Delta_{k}$ is the discriminant of $k$. Lemma 6.3. Let $\beta: \Gamma_{1} \rightarrow \Gamma_{2}$ be a homomorphism of metrized $R$-modules, and let $C>0$ be some constant such that the following conditions hold.

(i) the module $\Gamma_{1}$ is generated over $R$ by elements $\mathbf{y}_{1}, \ldots, \mathbf{y}_{\delta_{1}}$ satisfying

$$
\prod_{i=1}^{\delta_{1}} \prod_{\sigma \in B_{\infty}}\left\|\mathbf{y}_{i}\right\|_{\sigma} \leq C^{\delta_{1}}
$$

(ii) all nonzero $\gamma \in \Gamma_{2}$ satisfy $\prod_{\sigma \in B_{\infty}}\|\gamma\|_{\sigma} \geq C^{-1}$; and

(iii) for all $\gamma \in \Gamma_{1}, \prod_{\sigma \in B_{\infty}}\|\beta(\gamma)\|_{\sigma} \leq C \prod_{\sigma \in B_{\infty}}\|\gamma\|_{\sigma}$.

Then there exists a nonzero element $\gamma_{0} \in \operatorname{Ker} \beta$ with

$$
\prod_{\sigma \in B_{\infty}}\left\|\gamma_{0}\right\|_{\sigma} \leq\left(c_{1} C^{3}\right)^{\delta_{1} / \delta_{0}} e^{o\left(\delta_{1}^{2} / \delta_{0}\right)}
$$

for some constant $c_{1}=c_{1}(k)$.

Proof. This is a slight variation of [F 2, Proposition 2.18]. 
For this proof, it will be convenient to adopt the more common notation regarding infinite places; namely, $v$ varies over all archimedean absolute values and $k_{v}$ is the completion of $k$ at $v$, so $v$ corresponds to $\left[k_{v}: \mathbb{R}\right]$ places $\sigma \in B_{\infty}$. Then, if $\Gamma$ is a metrized, finitely generated lattice over $R$, and if $v$ corresponds to some $\sigma \in B_{\infty}$, let

$$
\|\gamma\|_{v}=|\gamma|_{\sigma}^{\left[k_{v}: \mathbb{R}\right]}, \quad \gamma \in \mathbb{R}
$$

so that

$$
\prod_{v \mid \infty}\|\gamma\|_{v}=\prod_{\sigma \in B_{\infty}}|\gamma|_{\sigma} .
$$

For all $v$, let the completion $\Gamma_{v}$ of $\Gamma$ at $v$ be given a measure such that, for each set $\gamma_{1}, \ldots, \gamma_{\delta}$ of $R$-linearly independent vectors in $\Gamma$ which generate $\Gamma$ modulo torsion, the product over all $v$ of the covolumes of the lattices generated over $\mathbb{Z}$ (if $k_{v} \cong \mathbb{R}$ ) or $\mathbb{Z}[i]$ (if $k_{v} \cong \mathbb{C}$ ) by $\gamma_{1}, \ldots, \gamma_{\delta}$ equals $\left(\Gamma: \sum_{i=1}^{\delta} R \gamma_{i}\right)$. Also let $V(\Gamma)$ be the product, over all $v$, of the volumes (relative to the above measures) of the unit balls (relative to $\|\cdot\|_{v}$ ) in $\Gamma_{v}$. Then, noting that the variant [V 1, 6.1.11] of Minkowski's theorem applies more generally to convex length functions, it follows that the successive minima $\lambda_{1}, \ldots, \lambda_{\delta}$ of $\Gamma$ relative to the length function $\prod_{v}\|\gamma\|_{v}$ satisfy the inequality

$$
c_{2}^{-\delta} e^{-o\left(\delta^{2}\right)} \leq \lambda_{1} \cdots \lambda_{\delta} V(\Gamma) \leq c_{3}^{\delta}
$$

where the constants $c_{2}$ and $c_{3}$ depend only on $k$.

In the current situation, let $\delta_{0}$ and $\delta_{2}$ be the ranks over $R$ of the kernel and image of $\beta$, respectively. By (i) and (ii), respectively,

$$
V\left(\Gamma_{1}\right) \geq\left(c_{2} C\right)^{-\delta_{1}} e^{-o\left(\delta_{1}^{2}\right)}
$$

and

$$
V(\operatorname{Im} \beta) \leq\left(c_{3} C\right)^{\delta_{2}}
$$

But by (iii),

$$
2^{[k: \mathbb{Q}] \delta_{0}} V(\operatorname{Ker} \beta) C^{\delta_{2}} V(\operatorname{Im} \beta) \geq V\left(\Gamma_{1}\right) .
$$

Thus,

$$
V(\operatorname{Ker} \beta) \geq 2^{-[k: \mathbb{Q}] \delta_{0}}\left(c_{2} C\right)^{-\delta_{1}}\left(c_{3} C^{2}\right)^{-\delta_{2}} e^{-o\left(\delta_{1}^{2}\right)}
$$

and the result follows from another application of Minkowski's theorem to the kernel of $\beta$.

The goal of this section, then, is to apply these two lemmas to the situation on $C^{n}$ and construct a small global section with certain properties. See Proposition 6.12 , below, for the exact statement. Before stating this proposition, however, we first introduce some notation and prove some lemmas to be used in the constructions of the proof. 
Isogenies and arithmetic. Let $J$ be the Jacobian of $C$. Fixing a point $P_{0} \in C$, let $j_{0}: C \rightarrow J$ be the map given by $P \mapsto \mathscr{O}\left(P-P_{0}\right)$. By [M, pp. 1011-1012],

$$
\operatorname{pr}_{i \ell}^{*} \mathscr{O}\left(\Delta-\left\{P_{0}\right\} \times \grave{C}-C \times\left\{P_{0}\right\}\right) \simeq \operatorname{pr}_{i}^{*} j_{0}^{*} \Theta+\operatorname{pr}_{\ell}^{*} j_{0}^{*} \Theta-\left(x_{i}+x_{\ell}\right)^{*} \Theta,
$$

where $\Theta$ is the divisor class of the theta divisor on $J$. But we need $\Theta$ to be a symmetric divisor class. Therefore fix $a \in J$ such that $(2 g-2) a=$ $\mathscr{O}\left((2 g-2) P_{0}-K_{C}\right)$, let $j(P)=j_{0}(P)+a$, and let $L$ be the class of the theta divisor defined relative to the map $j$. Then $L$ is a symmetric divisor class, and

$$
\Delta_{i \ell}^{\prime} \sim \operatorname{pr}_{i}^{*} j^{*} L+\operatorname{pr}_{\ell}^{*} j^{*} L-\left(x_{i}+x_{\ell}\right)^{*} L
$$

on $C^{n}$, up to $(2 g-2)$-torsion. See also [L 1, Chapter $5, \S 5$ ].

In the above paragraph, as in [F 2], the notation $\left(x_{i}+x_{\ell}\right)^{*} L$ means

$$
\left(\operatorname{pr}_{i} \times \operatorname{pr}_{\ell}\right)^{*}(j \times j)^{*} f^{*} L,
$$

where $f: J \times J \rightarrow J$ is the morphism defining addition on $J$. More generally, for $a, b \in \mathbb{Z}$ let $\left(a x_{i}+b x_{\ell}\right)^{*} L$ be defined similarly, where $f\left(x_{1}, x_{2}\right)=$ $a x_{1}+b x_{2}$, again using the group law on $J$. By the theorem of the cube, this expression is quadratic in $a$ and $b$, so by homogeneity it can be extended to $a, b \in \mathbb{Q}$. Thus, by the theorem of the cube,

$$
\left(\frac{1}{\sqrt{h_{i}}} x_{i} \pm \frac{1}{\sqrt{h_{\ell}}} x_{\ell}\right)^{*} L=\frac{\operatorname{pr}_{i}^{*} j^{*} L}{h_{i}}+\frac{\operatorname{pr}_{\ell}^{*} j^{*} L}{h_{\ell}} \mp \frac{\Delta_{i \ell}^{\prime}}{\sqrt{h_{i} h_{\ell}}} .
$$

Lemma 6.5 [F 2, Lemma 5.1]. Fix a model $\mathscr{J}$ for $J$ over $B$, an arithmetic divisor class $L$ on $\mathscr{J}$, and sections $\gamma_{1}, \ldots, \gamma_{m} \in \Gamma(\mathcal{J}, \mathscr{O}(L))$ which generate $\mathscr{O}(L)$ over the generic fibre. Then for all $a, b \in \mathbb{Z}$, not both zero, the linear equivalence

$$
\left(a x_{1}+b x_{2}\right)^{*} L \sim a^{2} x_{1}^{*} L+b^{2} x_{2}^{*} L-a b\left(x_{1}^{*} L+x_{2}^{*} L-\left(x_{1}+x_{2}\right)^{*} L\right)
$$

obtained via the theorem of the cube on $J$ turns all $\left(a x_{1}+b x_{2}\right)^{*} \gamma_{i}$ into sections

$$
\gamma_{i, a, b} \in \Gamma\left(W_{2}, \mathscr{O}\left(a^{2} x_{1}^{*} L+b^{2} x_{2}^{*} L-a b\left(x_{1}^{*} L+x_{2}^{*} L-\left(x_{1}+x_{2}\right)^{*} L\right)\right)\right)
$$

with the following properties:

(a) For all $i=1, \ldots, m$, for all places $\sigma \in B_{\infty}$, and for all $P \in C_{\sigma}^{2}$,

$$
\log \left\|\gamma_{i, a, b}(P)\right\| \leq c_{5, \sigma}\left(a^{2}+b^{2}\right)
$$

(b) For all places $\sigma \in B_{\infty}$ and all $P \in C_{\sigma}^{2}$,

$$
\max _{1 \leq i \leq m} \log \left\|\gamma_{i, a, b}(P)\right\| \geq-c_{5, \sigma}\left(a^{2}+b^{2}\right) .
$$

(c) For all non-archimedean places $v$ of $B$ and for all $i=1, \ldots, m$ the order of zero or pole of $\gamma_{i, a, b}$ along fibre components of $W_{2}$ over $v$ is bounded by $c_{5, v}\left(a^{2}+b^{2}\right)$. 
Moreover $c_{5, v}$ can be taken independent of $a$ and $b$, with $c_{5, v}=0$ for almost all $v$.

Proof. We may assume that $L$ is metrized with the metric whose curvature is invariant under translation. We may also assume that $C$ has a rational point $Q$; otherwise we may extend $B$, prove the lemma on the changed base, and then go back to $B$.

The linear equivalence (6.5.1) extends to a similar linear equivalence on some blowing-up of $W_{2}$, up to a divisor whose finite part is supported only on fibres over $B$ and whose infinite part is constant at each place (by the curvature assumption). We may assume that the intersection of this divisor with the closure of $(Q, Q)$ is an arithmetic divisor with multiplicities and Green's functions bounded quadratically in $a$ and $b$. This follows by properties of the canonical height of $Q$ and by finiteness of the class group. We use this linear equivalence to define $\gamma_{i, a, b}$, which then descends to a global section on $W_{2}$.

Then parts (a) and (b) are immediate consequences of the bound

$$
\log \left\|\gamma_{i}(P)\right\| \leq O(1) \quad \text { for all } i \text { and all } P \in J_{\sigma}
$$

and the bound

$$
\max _{1 \leq i \leq m} \log \left\|\gamma_{i}(P)\right\| \geq-O(1) \quad \text { for all } P \in J_{\sigma} .
$$

To prove (c), fix arithmetic divisors $D_{1}, D_{2}$, and $P$ on $W_{2}$, corresponding to $x_{1}^{*} L, x_{2}^{*} L$, and $\left(x_{1}+x_{2}\right)^{*} L$, respectively. We may assume that these are effective divisors along the generic fibre, and that they are not supported along any fibral component which meets the closure of $(Q, Q)$. The linear equivalence defining $\gamma_{i, a, b}$ then gives the equation of divisors

$$
\left(\gamma_{i, a, b}\right)=a(a-b) D_{1}+b(b-a) D_{2}+a b P+\left(f_{i, a, b}\right)
$$

where $f_{i, a, b}$ is a rational function on $W_{2}$. Let $G_{1}, G_{2}, G_{3}$, and $G_{i, a, b}$ be effective divisors, not supported along any fibre component, which coincide with $D_{1}, D_{2}, P$, and $\left(\gamma_{i, a, b}\right)$, respectively, up to fibre components. Then (6.5.2) becomes

(6.5.3) $G_{i, a, b}=a(a-b) G_{1}+b(b-a) G_{2}+a b G_{3}+F_{i, a, b}+\left(f_{i, a, b}\right)$,

where $F_{i, a, b}$ is a divisor supported only on fibre components. The lemma then reduces to showing that the multiplicities in $F_{i, a, b}+\left(f_{i, a, b}\right)$ of fibre components lying over non-archimedean places $v$ are bounded in absolute value by $c_{5, v}\left(a^{2}+b^{2}\right)$. At places of good reduction it is immediate that we can take $c_{5, v}=0$; at other places, the lemma is a consequence of the following combinatorial result.

Claim 6.5.4. Let $n_{1}, \ldots, n_{m}$ be integers and for $i=1, \ldots, m$ let $E_{i}$ be a closed cone in $\mathbb{R}^{n_{i}}$ with $E_{i} \cap\left(-E_{i}\right)=\{0\}$. Also, for $i=1, \ldots, m$ let $F_{i} \in \mathbb{R}^{n_{1}} \times \cdots \times \mathbb{R}^{n_{m}}$ be chosen such that

(i) $\sum_{i=1}^{m} F_{i}=0$;

(ii) For each nonempty subset $J \subsetneq\{1, \ldots, m\}$ and each $j=1, \ldots, m$, the $j$ th component of $\sum_{i \in J} F_{i}$ lies in $E_{j}$ if $j \notin J$ and $-E_{j}$ if $j \in J$, and there exists some $j \in J$ such that the $j$ th component is nonzero. 
Writing $E=E_{1} \times \cdots \times E_{m}$, fix $G_{j} \in E, j=1,2,3$. Assume $s_{1} \geq 0, s_{2} \geq 0$, $s_{3} \geq 0$, and $r_{1}, \ldots, r_{m} \in \mathbb{R}$ are given such that

$$
s_{1} G_{1}+s_{2} G_{2}+s_{3} G_{3}+\sum_{i=1}^{m} r_{i} F_{i} \in E .
$$

Then

$$
\max _{1 \leq i \leq m} r_{i}-\min _{1 \leq i \leq m} r_{i} \leq O\left(\max \left(s_{1}, s_{2}, s_{3}\right)\right),
$$

with the constant independent of $s_{1}, s_{2}, s_{3}$, and $r_{1}, \ldots, r_{m}$.

Proof. Assume it is false. Then by a compactness argument there exist real numbers $r_{1}, \ldots, r_{m}$, not all equal, such that

$$
E \ni \sum_{i=1}^{m} r_{i} F_{i}=\int_{\min r_{i}}^{\max r_{i}} \sum_{\left\{i \mid r_{i} \geq x\right\}} F_{i} d x ;
$$

we see that condition (ii) is contradicted for some index $j$ with $r_{j}=\max _{i} r_{i}$.

Now, to prove the lemma, let $C_{i}$ be the geometric components of a singular fibre, and let $F_{i}=m_{i} C_{i}$, where $m_{i}$ is the multiplicity of $C_{i}$ in the fibre. Let $\mathbb{R}^{n_{i}}$ be the subspace of $\operatorname{Pic}\left(C_{i}\right) \otimes_{\mathbb{Z}} \mathbb{R}$ generated by $G_{1}, G_{2}, G_{3}$, and $F_{1}, \ldots, F_{m}$. Let $E_{i}$ be the closure of the cone of effective divisors in $\mathbb{R}^{n_{i}}$. Then condition (i) of Claim 6.5.4 holds; also (ii) holds since the fibre is connected. Take $s_{1}=\max (0, a(a-b)), s_{2}=\max (0, b(b-a))$, and $s_{3}=\max (0, a b)$. Then (6.5.3) can be written

$$
\begin{aligned}
& G_{i, a, b}+\max (0, a(b-a)) G_{1}+\max (0, b(a-b)) G_{2}+\max (0,-a b) G_{3} \\
& =s_{1} G_{1}+s_{2} G_{2}+s_{3} G_{3}+\sum_{i=1}^{m} r_{i} F_{i}
\end{aligned}
$$

and the lemma then follows by applying the claim.

Remark. Faltings proves this result directly on $\mathscr{J} \times_{B} \mathscr{J}$. See [F 2], or also [V 6, Lemma 13.2].

The heights of linear constraints. The following two lemmas will lead to an upper bound on $h(A)$ in Lemma 6.2.

For these lemmas let $L^{\prime}$ be an arithmetic $\mathbb{Q}$-divisor class on $X$ which is ample on $C$. Then there exists a positive integer $d_{0}$ such that $d_{0} L^{\prime}$ is very ample on $C$. Let $\phi: C \rightarrow \mathbb{P}^{m}$ be the corresponding embedding, and let $N=$ $d_{0} \operatorname{deg} L^{\prime}$. The map $\phi$ may be changed by any automorphism of $\mathbb{P}^{m}$; therefore we may assume it to be suitably generic:

Assumption 6.6. The embedding $\phi$ is chosen such that the following properties hold:

(a) The curve $C$ does not meet the set $x_{0}=x_{1}=0$.

(b) The restriction to $C$ of the rational map $\mathbb{P}^{\mu} \rightarrow \mathbb{P}^{2}$ given by

$$
\left[x_{0}: \cdots: x_{\mu}\right] \mapsto\left[x_{0}: x_{1}: x_{2}\right]
$$


is birational onto its image. (Note that by part (a), the restriction to $C$ is everywhere defined.)

(c) None of the points in $\operatorname{Supp} D$ lies on the hyperplane $x_{0}=0$.

(d) None of the points in $\operatorname{Supp} D$ is a ramification point for the morphism $C \rightarrow \mathbb{P}^{1}$ defined by the rational function $x_{1} / x_{0}$.

Lemma 6.7. There exist sections $\gamma_{0}, \gamma_{1}, \gamma_{2} \in \Gamma\left(X, \mathscr{O}\left(d_{0} L^{\prime}\right)\right)$ such that

(i) their restrictions to $C$ are equal to constant multiples of the sections $\phi^{*} x_{0}, \phi^{*} x_{1}, \phi^{*} x_{2}$, respectively; and

(ii) the sections

$$
\gamma_{0}^{d / d_{0}-u-w} \gamma_{1}^{u} \gamma_{2}^{w}, \quad 0 \leq u \leq d / d_{0}-w, \quad 0 \leq w<N
$$

form a basis over $R$ of a submodule

$$
\Gamma_{1}(d) \subseteq \Gamma\left(X, d L^{\prime}\right)
$$

such that the rank (over $R$ ) of the quotient module is bounded independently of $d$, and such that the torsion in the quotient is annihilated by an integer bounded by $c^{d}$, for a constant $c$ independent of $d$.

Proof. By parts (a) and (b) of Assumption 6.6, the sections

$$
\left.\left(x_{0}^{d / d_{0}-u-w} x_{1}^{u} x_{2}^{w}\right)\right|_{C}, \quad 0 \leq u \leq d / d_{0}-w, \quad 0 \leq w<N
$$

are linearly independent over $k$, and generate a subspace of $\Gamma\left(C, \mathscr{O}\left(d L^{\prime}\right)\right)$ of dimension $d \operatorname{deg} L^{\prime}-N(N-3) / 2$, if $d / d_{0} \geq N-2$. By Riemann-Roch, $h^{0}\left(C, d L^{\prime}\right)=d \operatorname{deg} L^{\prime}+1-g$ for $d$ large; this implies the assertion about the rank of the quotient.

To obtain the sections $\gamma_{i}$, take the closures of $\phi^{*} x_{i}$ in $\Gamma\left(X, \mathscr{O}\left(L^{\prime}\right)\right)$ and clear denominators. Then the assertion about the $R$-rank of the quotient follows from the previous paragraph. The bound on the annihilator of the torsion in the quotient is a consequence of the fact that the closure of $\phi(C)$ in $\mathbb{P}_{B}^{m}$ is noetherian, together with the fact that the sections (6.7.2) generate all of $\bigoplus_{i \geq 0} \Gamma(\phi(C), \mathscr{O}(i))$ (injecting $\mathscr{O}(i)$ into $\mathscr{O}(i+1)$ via the section $\left.x_{0}\right)$.

For the next lemma, the height of a linear constraint relative to a given basis is defined to be the height of its vector of coefficients, relative to that basis.

Lemma 6.8. Fix $Q \in \operatorname{Supp} D$ and let $L^{\prime}$ and $d_{0}$ be as before. Let $h_{1}, \ldots, h_{n}$ be positive rational numbers, and let $d$ be a positive integer such that $d / h_{i} \in d_{0} \mathbb{Z}$ for all $i$. Consider the space $\otimes_{i=1}^{n} \Gamma_{1}\left(d / h_{i}\right)$, endowed with the basis obtained from the basis (6.7.1) on each factor. Then the subspace of $\otimes_{i=1}^{n} \Gamma_{1}\left(d / h_{i}\right)$ corresponding to sections having a prescribed truncated index function at $(Q, \ldots, Q)$ relative to multiplicities $d_{i}:=d x / h_{i}$ is cut out by linear constraints of height $\leq c_{6} d \sum_{i} 1 / h_{i}$ for some constant $c_{6}$ independent of $d$ and $h_{1}, \ldots, h_{n}$.

Proof. We first reduce to the case $n=1$. Indeed, the general case follows by induction on $n$, writing $\otimes_{i=1}^{n} \Gamma_{1}\left(d / h_{i}\right)$ in terms of the chosen basis for $\Gamma_{1}\left(d / h_{n}\right)$ with coefficients in $\bigotimes_{i=1}^{n-1} \Gamma_{1}\left(d / h_{i}\right)$. 
Since $n=1$, we may assume that $h_{1}=1$. Also, the notion of index function reduces to a lower bound on the order of vanishing at $Q$.

By Assumption 6.6(c), the section $\gamma_{0}$ is nonzero at $Q$. Then given any $\gamma \in \Gamma_{1}(d)$, the function $\gamma / \gamma_{0}^{d / d_{0}}$ is a rational function which is regular at $Q$. Writing it as a power series $a_{0}+a_{1} \xi+\cdots$ in terms of some local parameter $\xi$ at $Q$, we see that $\gamma$ vanishes to order $\geq i$ at $Q$ if and only if $a_{j}=0$ for all $j=0, \ldots, i-1$. It will suffice to find a local parameter $\xi$ such that the corresponding constraints $a_{j}=0$ have the desired height bound relative to the basis (6.7.1). To get the coefficients of these constraints, we expand $\left(\gamma_{1} / \gamma_{0}\right)^{u}\left(\gamma_{2} / \gamma_{0}\right)^{w}$ as power series in $\xi$.

First, however, note that the divisor $\left(\gamma_{i}\right)-\left(x_{i}\right)$ is a Cartier divisor on the closure of the graph of $\phi$ in $\mathbb{P}_{X}^{m}$ which is supported only on fibres over $B$. Then, since $\left(\gamma_{i} / \gamma_{0}\right)$ and $\left(x_{i} / x_{0}\right)$ are principal divisors, the corresponding functions differ by a factor in $k$. Thus, it will suffice to expand the functions $\left(x_{1} / x_{0}\right)^{u}\left(x_{2} / x_{0}\right)^{w}$ as power series in $\xi$ and prove bounds on the coefficients of those series.

We first consider the local parameter $\xi$. This will be chosen so that there exist constants $c_{7, v}$ and $c_{8, v}$ for all places $v$ of $k$, with $c_{7, v}=c_{8, v}=1$ for almost all $v$, such that one has a power series

$$
\frac{x_{1}}{x_{0}}=a_{0}+a_{1} \xi+a_{2} \xi^{2}+\cdots
$$

with $\left|a_{i}\right|_{v} \leq c_{7, v} c_{8, v}^{i}$ for all $v$ and all $i \geq 0$, and likewise for the function $x_{2} / x_{0}$.

Let $C^{\prime}$ denote the image of $C$ in $\mathbb{P}^{2}$ via the map taking $\left[x_{0}: \cdots: x_{\mu}\right]$ to $\left[x_{0}: x_{1}: x_{2}\right]$. Also let $\mathbb{A}^{2}=\mathbb{P}^{2} \backslash\left\{x_{0}=0\right\} \cong \operatorname{Spec} k\left[x_{1} / x_{0}, x_{2} / x_{0}\right]$. Then $C^{\prime} \cap \mathbb{A}^{2}$ is the set of solutions of $f_{0}\left(x_{1} / x_{0}, x_{2} / x_{0}\right)=0$ for some polynomial $f_{0}$; this can be rewritten $f(\xi, \eta)=0$, where

$$
\xi=x_{1} / x_{0}-\left(x_{1} / x_{0}\right)(Q) \quad \text { and } \quad \eta=x_{2} / x_{0}-\left(x_{2} / x_{0}\right)(Q) \text {. }
$$

Note that by Assumption 6.6(c), $Q \in \mathbb{A}^{2}$, so that the expressions for $\xi$ and $\eta$ make sense. We take $\xi$ as the local parameter; then obviously the coefficients of $x_{1} / x_{0}$, written as a power series in $\xi$, satisfy the desired growth conditions. We need to show that the same is true for $x_{2} / x_{0}$, which is equivalent to showing the same for $\eta$.

Since $Q \in C$, the constant term of $f$ is zero; by Assumption 6.6(d), the coefficient of $\eta$ in $f$ is nonzero. Then, by the Implicit Function Theorem for analytic functions, the growth condition for the coefficients of $\eta$ holds at the archimedean places; by the $p$-adic equivalent of this theorem, the same holds for non-archimedean $v$ as well. Clearing denominators, we may assume that all coefficients of $f$ are algebraic integers. Then, by solving explicitly for each coefficient of $\eta$, it follows that one can take $c_{7, v}=c_{8, v}=1$ for all non-archimedean $v$ satisfying

$$
\left|\left(x_{1} / x_{0}\right)(Q)\right|_{v}=\left|\left(x_{2} / x_{0}\right)(Q)\right|_{v}=\left|e_{1}\right|_{v}=1,
$$

where $e_{1}$ is the coefficient of $\eta$ in $f$. 
We now claim that for all places $v$ of $k$ there exist constants $c_{9, v}$ such that $c_{9, v}=1$ for almost all $v$, and having the following property. For all natural numbers $u, w$, and $j$, the coefficient $f_{j}$ of $\xi^{j}$ in $\left(x_{1} / x_{0}\right)^{u}\left(x_{2} / x_{0}\right)^{w}$ satisfies

$$
\left|f_{j}\right|_{v} \leq c_{9, v}^{\max (u+w, j, 1)} .
$$

Indeed, write $x_{1} / x_{0}=a_{0}+a_{1} \xi+a_{2} \xi^{2}+\cdots$ and $x_{2} / x_{0}=b_{0}+b_{1} \xi+b_{2} \xi^{2}+\cdots$. The $f_{j}$ can be written as a polynomial in the $a_{i}$ and $b_{i}$ with positive integral coefficients. This immediately implies the result for non-archimedean places $v$, since we can take $c_{9, v}=c_{7, v} c_{8, v}$. At archimedean places, we take $c_{9, v}=$ $4 c_{7, v} c_{8, v}$, using the fact that the sum of the coefficients is given by

$$
\left(\begin{array}{c}
u+w+j-1 \\
j
\end{array}\right) \leq 2^{u+w+j} \leq 4^{\max (u+w, j)} .
$$

Partial derivatives. Let $\gamma$ be a section of a metrized line sheaf $\mathscr{L}$ on $X$, and let $P \in C(k)$, corresponding to a section $s: B \rightarrow X$. Then for integers $i \geq 0$ we shall now define the $i$ th "derivative" $D_{i} \gamma(P)$ as a section

$$
D_{i} \gamma \in \Gamma\left(B, s^{*}\left(\mathscr{L} \otimes \omega_{X / B}^{\otimes i}\right)\right),
$$

provided that $D_{j} \gamma=0$ for all $j=0, \ldots, i-1$. Fix a local section $\gamma_{0}$ of $\mathscr{L}$ which has neither a zero nor a pole at $P$; then

$$
\gamma=\gamma_{0} \sum_{i \geq 0} a_{i} z^{i}
$$

and $\gamma_{0} a_{i} z^{i}$ lies in the subsheaf

$$
\mathscr{L} \otimes \mathscr{O}(-i P) \subseteq \mathscr{L} \text {. }
$$

Its restriction to the image of $s$ is well-defined, independent of the choices of $z$ and $\gamma_{0}$, by the assumption on $i$. Using different local parameters $z$, it follows that this section is a well-defined element of the sheaf $s^{*}(\mathscr{L} \otimes \mathscr{O}(-i P)) \cong$ $s^{*}\left(\mathscr{L} \otimes \omega_{X / B}^{\otimes i}\right)$ at every closed point of $B$.

Partial derivatives on $W$ are defined similarly.

An immediate consequence of the above definition is that at all nonarchimedean places, the partial derivative is a regular section of the indicated sheaf. Corresponding statements will also be needed at archimedean places; these take the form of upper bounds on metrics. These will ultimately be used to satisfy Condition (ii) of Lemma 6.3.

By a compactness argument there exists a positive constant $\rho=\rho(C)$ such that for all $\sigma \in B_{\infty}$ and all $P \in C_{\sigma}$ there exists a neighborhood $U_{P} \subseteq C$ of $P$ with coordinate $z_{P}: U_{P} \stackrel{\sim}{\rightarrow} \mathbb{D}_{\rho}$ such that $z_{P}(P)=0$ and such that the inequality

$$
d d^{c}|z|^{2} \leq \omega
$$

holds on $U_{P}$. Here $\omega$ is defined as the admissible metric on the tangent bundle; see (6.9.1) below. If $P \in C(\bar{k})$ and $\sigma: k(P) \hookrightarrow \mathbb{C}$, then we also write $U_{P, \sigma}$ and $z_{P, \sigma}$ in place of $U_{P_{\sigma}}$ and $z_{P_{\sigma}}$, respectively. 
Lemma 6.9. For any $P^{\prime} \in U_{P}$, the norm at $P^{\prime}$ of the local section $z_{P}-z_{P}\left(P^{\prime}\right)$ of $\mathscr{O}\left(-P^{\prime}\right)$ satisfies

$$
\left\|\left(z_{P}-z_{P}\left(P^{\prime}\right)\right)\left(P^{\prime}\right)\right\| \leq 1 \text {. }
$$

Proof. Writing $G_{\sigma}\left(P^{\prime}, P^{\prime \prime}\right)=f\left(P^{\prime \prime}\right)\left|z_{P}\left(P^{\prime \prime}\right)-z_{P}\left(P^{\prime}\right)\right|^{2}$ for some positive function $f$ gives

$$
\omega\left(P^{\prime}\right)=\left.d_{P^{\prime \prime}} d_{P^{\prime \prime}}^{c} G_{\sigma}\left(P^{\prime}, P^{\prime \prime}\right)\right|_{P^{\prime \prime}=P^{\prime}}=f\left(P^{\prime}\right) d d^{c}|z|^{2} .
$$

The assumption $\omega \geq d d^{c}|z|^{2}$ implies that $f\left(P^{\prime}\right) \geq 1$. Then

$$
\left\|\left(z_{P}-z_{P}\left(P^{\prime}\right)\right)\left(P^{\prime}\right)\right\|^{2}=\lim _{P^{\prime \prime} \rightarrow P^{\prime}} \frac{\left|z_{P}\left(P^{\prime \prime}\right)-z_{P}\left(P^{\prime}\right)\right|^{2}}{G_{\sigma}\left(P^{\prime}, P^{\prime \prime}\right)}=\frac{1}{f\left(P^{\prime}\right)} \leq 1 .
$$

Lemma 6.10. Fix $\sigma \in B_{\infty}$ and a point $P_{0}=\left(P_{1}, \ldots, P_{n}\right) \in C_{\sigma}^{n}$. Let $\mathscr{L}$ be a metrized line sheaf on $W$, and let $\gamma \in \Gamma(W, \mathscr{L})$. Let $\gamma_{0}$ be a section of $\mathscr{L}$ on $U:=U_{P_{1}} \times \cdots \times U_{P_{n}}$ which is nonzero at $P_{0}$. Suppose $(j)=\left(j_{1}, \ldots, j_{n}\right)$ is a tuple for which $D_{(j)} f^{*} \gamma\left(P_{1}, \ldots, P_{n}\right)$ is defined. Then

$$
-\log \left\|D_{(j)} \gamma\left(P_{0}\right)\right\| \geq-\log \|\gamma\|_{\text {sup }, \sigma}+\sum_{i=1}^{n} j_{i} \log \rho+\log \inf _{P \in U} \frac{\left\|\gamma_{0}(P)\right\|}{\left\|\gamma_{0}\left(P_{0}\right)\right\|} .
$$

Proof. Writing $z_{P_{0}}$ for the tuple of functions $\left(\mathrm{pr}_{1}^{*} z_{P_{1}}, \ldots, \mathrm{pr}_{n}^{*} z_{P_{n}}\right)$ and writing

$$
\gamma=\gamma_{0} \sum_{(i) \geq 0} a_{(i)} z_{P_{0}}^{(i)}
$$

gives

$$
\begin{aligned}
\|\gamma\|_{\text {sup }, \sigma} & \geq \sup _{P \in U}\left\|\gamma_{0}(P)\right\|\left|\sum_{(i) \geq 0} a_{(i)} z_{P_{0}}^{(i)}\right| \\
& \geq \inf _{P \in U}\left\|\gamma_{0}(P)\right\| \sup _{z \in \mathbb{D}_{\rho}^{n}}\left|\sum_{(i) \geq 0} a_{(i)} z^{(i)}\right| \\
& \geq\left\|\gamma_{0}\left(P_{0}\right)\right\| \inf _{P \in U} \frac{\left\|\gamma_{0}(P)\right\|}{\left\|\gamma_{0}\left(P_{0}\right)\right\|}\left|a_{(j)}\right| \rho^{j_{1}+\cdots+j_{n}}
\end{aligned}
$$

by the multivariable Cauchy integral formula

$$
a_{(j)}=\int_{\left|z_{n}\right|=\rho} \cdots \int_{\left|z_{1}\right|=\rho} \frac{\sum_{(i)} a_{(i)} z^{(i)}}{z_{1}^{j_{1}+1} \cdots z_{n}^{j_{n}+1}} \frac{d z_{1} \cdots d z_{n}}{(2 \pi \sqrt{-1})^{n}} .
$$

Thus by Lemma 6.9,

$$
\left\|\left(\gamma_{0} a_{(j)} z_{P_{0}}^{(j)}\right)\left(P_{0}\right)\right\| \rho^{j_{1}+\cdots+j_{n}} \inf _{P \in U} \frac{\left\|\gamma_{0}(P)\right\|}{\left\|\gamma_{0}\left(P_{0}\right)\right\|} \leq\|\gamma\|_{\text {sup }, \sigma} .
$$

Corollary 6.11. Fix an arithmetic divisor $L^{\prime \prime}$ on $X$. Then for all sufficiently divisible $d$ and all nonzero sections $\gamma \in \Gamma\left(W, \mathscr{O}\left(\sum_{i} d \operatorname{pr}_{i}^{*} L^{\prime \prime} / h_{i}\right)\right)$,

$$
\sum_{\sigma \in B_{\infty}}-\log \|\gamma\|_{\mathrm{sup}, \sigma} \leq c_{11} d \sum_{i} 1 / h_{i}
$$

for some constant $c_{11}=c_{11}\left(L^{\prime \prime}\right)$. 
Proof. The assertion is unaffected (up to changing $c_{11}$ ) by base change; therefore we may assume that $C$ has a rational point $Q$. Fix a local holomorphic section $\gamma_{00}$ of $\mathscr{O}\left(L^{\prime \prime}\right)$ which does not vanish on $\bar{U}_{Q, \sigma}$. Let

$$
c_{10}=-\log \min _{\sigma: k \rightarrow \mathbb{C}} \inf _{P \in U_{Q, \sigma}} \frac{\left\|\gamma_{00}(P)\right\|}{\left\|\gamma_{00}\left(Q_{\sigma}\right)\right\|}
$$

and let $\gamma_{0}=\prod_{i} \operatorname{pr}_{i}^{*} \gamma_{00}^{d / h_{i}}$, so that

$$
\frac{\left\|\gamma_{0}\left(P_{1}, \ldots, P_{n}\right)\right\|}{\left\|\gamma_{0}\left(Q_{\sigma}, \ldots, Q_{\sigma}\right)\right\|} \geq \exp \left(-c_{10} d \sum_{i} 1 / h_{i}\right)
$$

for all $\left(P_{1}, \ldots, P_{n}\right) \in U_{Q, \sigma}^{n}$ and all $\sigma: k \hookrightarrow \mathbb{C}$.

Let $(j)=\left(j_{1}, \ldots, j_{n}\right)$ be a multi-index such that $D_{(j)} \gamma(Q, \ldots, Q)$ is defined and nonzero. Then by (6.10.1), for all $\sigma$

$(6.11 .1)-\log \left\|D_{(j)} \gamma\left(Q_{\sigma}, \ldots, Q_{\sigma}\right)\right\| \geq-\log \|\gamma\|_{\text {sup }, \sigma}+\sum_{i=1}^{n} j_{i} \log \rho-c_{10} d \sum_{i=1}^{n} \frac{1}{h_{i}}$.

But since $D_{(j)} \gamma(Q, \ldots, Q)$ is an integral section of

$$
\mathscr{O}\left(\sum_{i} d \mathrm{pr}_{i}^{*} L^{\prime \prime} / h_{i}-\sum_{i} j_{i} \mathrm{pr}_{i}^{*} Q\right)
$$

and $j_{i} \leq\left(d / h_{i}\right) \operatorname{deg} L^{\prime \prime}$,

$$
\sum_{\sigma}-\log \left\|D_{(j)} \gamma\left(Q_{\sigma}, \ldots, Q_{\sigma}\right)\right\| \leq \sum_{i}\left(d / h_{i}\right) \operatorname{deg} s_{Q}^{*}\left(\mathscr{O}\left(L^{\prime \prime}\right) \otimes \omega_{X / B}^{\otimes \operatorname{deg} L^{\prime \prime}}\right)
$$

where $s_{Q}: B \rightarrow X$ is the section corresponding to $Q$. The desired estimate then follows by summing (6.11.1) and using the upper bound on the $j_{i}$.

Construction of a global section. We are now ready to prove the main result of this section.

Proposition 6.12. There exists a constant $c_{4}=c_{4}(X, D, n, x)$ such that for all tuples $(h)$ satisfying (4.1) and (4.4.1), and all integers $d$ sufficiently divisible (depending also on $(h)$ ), there exists a section $\gamma$ of $\mathscr{O}\left(d Y_{x,(h)}\right)$ such that:

(i) the sup norms $\|\gamma\|_{\text {sup }, \sigma}$ of $\gamma$ at all $\sigma \in B_{\infty}$ satisfy the inequality

$$
\sum_{\sigma \in B_{\infty}}-\log \|\gamma\|_{\text {sup }, \sigma} \geq-c_{4} d \sum_{i=1}^{n} 1 / h_{i}
$$

and

(ii) the section has index function $\# I\left((\# I+1) / 2 n-\varepsilon_{3}\right)$ relative to $d_{i}:=d x / h_{i}$ at $(Q, \ldots, Q)$ for all $Q \in \operatorname{Supp} D$.

Proof. Let $\gamma_{1}, \ldots, \gamma_{m}$ be sections in $\Gamma(\mathscr{J}, \mathscr{O}(L))$ that generate $\mathscr{O}(L)$ over $J$. Then the sections

$$
\prod_{i<\ell}\left(\frac{1}{\sqrt{h_{i}}} x_{i} \pm \frac{1}{\sqrt{h_{\ell}}} x_{\ell}\right)^{*} \gamma_{j_{i \ell}}^{d}
$$


as $\left(j_{i \ell}\right)$ varies over all tuples in $\{1, \ldots, m\}^{n(n-1) / 2}$, give a Čech-like complex

$$
\begin{aligned}
& 0 \rightarrow \Gamma\left(W, \mathscr{O}\left(d Y_{x,(h)}\right)\right) \stackrel{\alpha}{\rightarrow} \Gamma\left(W, \mathscr{O}\left(\sum_{i} d \operatorname{pr}_{i}^{*} L^{\prime} / h_{i}\right)\right)^{a} \\
& \quad \stackrel{\beta}{\rightarrow} \Gamma\left(W, \mathscr{O}\left(\sum_{i} d \mathrm{pr}_{i}^{*} L^{\prime \prime} / h_{i}\right)\right)^{b}
\end{aligned}
$$

up to certain denominators. Here $a=m^{n(n-1) / 2}, \quad b=\left(\begin{array}{l}a \\ 2\end{array}\right), L^{\prime}=x F+$ $2(n-1) j^{*} L$, and $L^{\prime \prime}=x F+4(n-1) j^{*} L$. By Lemma 6.5(c) we may clear denominators by multiplying by integers bounded by $\exp \left(c d \sum_{i} 1 / h_{i}\right)$, and the resulting sequence will be exact up to torsion annihilated by integers of a comparable size. Throughout this proof, these constants $c$ will depend on $x, n$, and $\gamma_{1}, \ldots, \gamma_{m}$, but not on $d$ or $(h)$.

At places $\sigma \in B_{\infty}$ we place norms on the terms of (6.12.2) by using the sup norm on the first term, and the largest sup norm of each element in the tuple for the second and third terms. It follows from Lemma 6.5(b) that the map $\alpha$ decreases norms by a factor of at most $\exp \left(-c d \sum_{i} 1 / h_{i}\right)$. Likewise, Lemma 6.5 (a) implies that $\beta$ can increase norms by at most a factor of $\exp \left(c d \sum_{i} 1 / h_{i}\right)$. The operations of clearing denominators in the previous paragraph do not affect the shape of these bounds.

The remainder of the proof takes place in three steps.

(1) Pass to a sublattice of $\Gamma_{1}$ (and $\Gamma_{0}$ ) as in Lemma 6.7.

(2) Pass to the smaller sublattice of sections of $\Gamma_{1}$ which have the given truncated index function at $(Q, \ldots, Q)$ for all $Q \in \operatorname{Supp} D$.

(3) Apply Lemma 6.3 to obtain a suitable element of the kernel of $\alpha$.

Then, by Lemma 5.3, this element will have the desired index function at all $(Q, \ldots, Q)$.

Step 1. Replace the second term of $(6.12 .2)$ with the $R$-module

$$
\Gamma_{1}^{\prime}:=\left(\bigotimes_{i=1}^{n} \Gamma_{1}\left(d / h_{i}\right)\right)^{a},
$$

as in Lemma 6.7, where $\Gamma_{1}\left(d / h_{i}\right)$ is identified with a subgroup of $\Gamma\left(W, d \operatorname{pr}_{i}^{*} L^{\prime} / h_{i}\right)$ as in that lemma. This requires us to replace the first factor with a submodule $\Gamma_{0}^{\prime}$. However, by Lemma 6.7, the codimension of $\Gamma_{0}^{\prime}$ is at most

$$
\operatorname{codim} \Gamma_{1}^{\prime} \leq O\left(d^{n-1}\right)
$$

with the constant independent of $d$. Again we must cancel denominators, but by part (ii) of the lemma this does not invalidate the previous estimates on the torsion of cohomology and effect of $\alpha$ and $\beta$ on norms at infinite places. As a basis for $\Gamma_{1}^{\prime}$ we use the basis obtained from (6.7.1); then norms of elements of this basis will be bounded by $\exp \left(c d \sum_{i} 1 / h_{i}\right)$. From the above estimates it follows that given any element $\gamma_{1} \in \Gamma_{1}^{\prime}$ lying in the kernel of $\beta$, there exists some section $\gamma \in \Gamma_{0}^{\prime}$ such that $\alpha(\gamma)$ equals a multiple of $\gamma_{1}$, and such that for all $\sigma \in B_{\infty}$,

$$
-\log \|\gamma\|_{\text {sup }, \sigma} \geq-\log \left\|\gamma_{1}\right\|_{\text {sup }, \sigma}-c d \sum_{i} 1 / h_{i}
$$


Step 2. By Lemma 6.8 , the condition that a section in $\bigotimes_{i=1}^{n} \Gamma_{1}\left(d / h_{i}\right)$ has a certain truncated index function at $(Q, \ldots, Q)$ for some $Q \in \operatorname{Supp} D$ is determined by linear conditions of height at most $c d \sum_{i} 1 / h_{i}$. For each $Q$ we only apply these conditions to one of the direct summands of $\Gamma_{1}^{\prime}$. This gives a submodule $\Gamma_{1}^{\prime \prime}$. Applying Siegel's lemma, we note that (i) the ranks of $\Gamma_{1}^{\prime \prime}$ and $\Gamma_{1}^{\prime}$ are of the same order of magnitude; (ii) the height of a matrix is bounded by the sum of the heights of its rows plus $\log (\mathscr{M} !)$; and (iii) in this case $\mathscr{M}$ grows like $d^{n}$ (fixing everything but $d$ ), so that the term $\log (\mathscr{M} !)$ is $o\left(d^{n+1}\right)$. Thus by Lemma 6.2 and (5.6.1) there exists a basis $\mathbf{y}_{1}, \ldots, \mathbf{y}_{\delta_{1}}$ (up to torsion) for $\Gamma_{1}^{\prime \prime}$ such that

$$
\sum_{i=1}^{\delta_{1}} \sum_{\sigma \in B_{\infty}}-\log \left\|\mathbf{y}_{i}\right\|_{\text {sup }, \sigma} \geq \frac{\varepsilon_{2}}{2} \prod_{i=1}^{n}\left(d_{i}+1\right) \cdot c d \sum_{i} 1 / h_{i}-o\left(d^{n+1}\right) .
$$

Again, we must shrink $\Gamma_{0}^{\prime}$ accordingly, obtaining a submodule $\Gamma_{0}^{\prime \prime}$ of rank

$$
\delta_{0} \geq \frac{\varepsilon_{2}}{2} \frac{d^{n} x^{n}}{h_{1} \cdots h_{n}}-O\left(d^{n-1}\right)
$$

by Proposition 5.6 and (6.12.3).

Step 3. To apply Lemma 6.3, use

$$
\begin{aligned}
\delta_{1} & =\prod_{i=1}^{n}\left(d \operatorname{deg} L^{\prime} / h_{i}-N(N-3) / 2\right) \\
& \leq \frac{d^{n} x^{n}}{h_{1} \cdots h_{n}}+O\left(d^{n-1}\right)
\end{aligned}
$$

and

$$
C=\exp \left(c d \sum_{i} 1 / h_{i}\right) .
$$

Condition (i) of the lemma holds by (6.12.5); (ii), by Corollary 6.11 ; and (iii) holds by construction and Lemma 6.5. Thus, by Lemma 6.3 , there exists a nonzero element $\gamma \in \Gamma_{0}$ such that $\alpha(\gamma)$ satisfies (6.12.1). By (6.12.4), then, so does $\gamma$.

\section{Choosing the Algebraic PoINTs}

For points $P \in C(\bar{k})$, recall that $H_{P}$ is defined as the corresponding horizontal prime Arakelov divisor on $X$. Let $\nu_{P}=\operatorname{deg} H_{P}$, and let

$$
E_{P}=\frac{H_{P}}{\nu_{P}}
$$

so that $\operatorname{deg} E_{P}=1$. By the definition of $d_{\mathrm{a}}(P)$,

$$
d_{\mathrm{a}}(P)=\frac{\nu_{P}\left(E_{P}^{2}\right)+\left(E_{P} \cdot \omega_{X / B}\right)}{[k: \mathbb{Q}]} .
$$


By Lemma 2.2 and (2.3), we are considering only points $P$ for which

$$
\nu_{P}=\nu
$$

and

$$
d_{\mathrm{a}}(P) \leq m(D, P)+h_{K}(P)-\varepsilon h(P) .
$$

For points satisfying (7.2) and (7.3), (7.1) gives

$$
\frac{\nu\left(E_{P}^{2}\right)}{[k: \mathbb{Q}]} \leq m(D, P)-\varepsilon h(P) .
$$

Proposition 7.5. Let $\varepsilon>0, \varepsilon^{\prime}>0, \varepsilon_{4}>0$, and $\varepsilon_{5}>0$ be given, satisfying

$$
\nu \# S \varepsilon_{4}+2 \nu \varepsilon_{5}+2 \nu \varepsilon^{\prime}<\varepsilon .
$$

Then there exist constants $c_{12}$ and $h_{\min , 0}$, depending only on $X, D, S, \nu$, $\varepsilon, \varepsilon^{\prime}, \varepsilon_{4}$, and $\varepsilon_{5}$, having the following property. Let $\mathscr{P}$ be a set of points $P \in C(\bar{k})$ satisfying (7.2) and (7.3), and such that the fields $k(P)$ are linearly disjoint over $k$. Then there exists a partitioning of $\mathscr{P}$ into finitely many subsets such that for any such subset $\mathscr{P}^{\prime}$, we have:

(a) there exist constants $\kappa_{v, \alpha}$ and points $Q_{v, \alpha} \in \operatorname{Supp} D$ for all $v \in S$ and all $\alpha=1, \ldots, \nu$ such that

$$
0 \leq \kappa_{v, \alpha}<\operatorname{deg} D+2 \varepsilon_{4},
$$

and for all $P \in \mathscr{P}^{\prime}$ with $h(P) \geq h_{\min , 0}$ there exists a permutation $P^{[1]}, \ldots, P^{[\nu]}$ of the conjugates of $P$ in $C\left(\bar{k}_{v}\right)$, such that

$$
\frac{g_{v}\left(P^{[\alpha]}, D\right)}{\nu[k: \mathbb{Q}]} \geq \kappa_{v, \alpha} h(P)
$$

and

$$
g_{v}\left(P^{[\alpha]}, Q_{v, \alpha}\right)=\max _{Q \in \operatorname{Supp} D} g_{v}\left(P^{[\alpha]}, Q\right)
$$

for all $v \in S$ and all $\alpha=1, \ldots, \nu$.

(b) For all tuples $\left(P_{1}, \ldots, P_{n}\right)$ of distinct points in $\mathscr{P}^{\prime}$ with $h\left(P_{i}\right) \geq h_{\min , 0}$ for all $i$, for all $\eta>0$, and for all tuples $(h)=\left(h_{1}, \ldots, h_{n}\right)$ satisfying (4.1) and the condition

$$
h_{i} \leq h\left(P_{i}\right) \leq h_{i}(1+\eta), \quad i=1, \ldots, n,
$$

the arithmetic curve $s: E \rightarrow X \times_{B} \cdots \times_{B} X$ corresponding to the point $\left(P_{1}, \ldots, P_{n}\right) \in C^{n}(\bar{k})$ satisfies the inequality

$$
\begin{aligned}
& \frac{\operatorname{deg} s^{*} Y_{x,(h)}}{\left[k\left(P_{1}, \ldots, P_{n}\right): \mathbb{Q}\right]} \\
& \quad \leq n\left(x(1+\eta)-(n-1)\left(1+\varepsilon^{\prime}-\frac{\sum \kappa_{v, \alpha}}{2 \nu}\right)\right)+c_{12} \sum_{i<\ell} \frac{1}{\sqrt{h_{i} h_{\ell}}} .
\end{aligned}
$$


Proof. There exists a constant $c$ such that for all $P \in C(\bar{k})$ and all $v \in S$,

$$
\frac{\left(E_{P} \cdot D\right)_{v}}{[k: \mathbb{Q}]} \leq \frac{\left(E_{P} \cdot D\right)}{[k: \mathbb{Q}]}+c .
$$

Also, by the property of heights relative to numerically equivalent divisors [V 1 , 1.2.9d], for all $P \in C(\bar{k})$ of sufficiently large height,

$$
\frac{\left(E_{P} \cdot D\right)}{[k: \mathbb{Q}]}<\left(\operatorname{deg} D+\varepsilon_{4}\right) h(P) .
$$

Therefore, there exists a constant $h_{0}=h_{0}\left(X, D, S, \varepsilon_{4}\right)$ such that if $P \in C(\bar{k})$ and $h(P) \geq h_{0}$, then

$$
\frac{\left(E_{P} \cdot D\right)_{v}}{[k: \mathbb{Q}]}<\left(\operatorname{deg} D+2 \varepsilon_{4}\right) h(P)
$$

for all $v \in S$. Hence let $\kappa_{v, \alpha}$ range over all of the tuples of integer multiples of $\varepsilon_{4}$ in the range $0 \leq \kappa_{v, \alpha}<\operatorname{deg} D+2 \varepsilon_{4}$. For each $P \in C(\bar{k})$ with $h(P)>h_{0}$, we may find such a tuple with

$$
\kappa_{v, \alpha} h(P) \leq \frac{g_{v}\left(P^{[\alpha]}, D\right)}{\nu[k: \mathbb{Q}]} \leq\left(\kappa_{v, \alpha}+\varepsilon_{4}\right) h(P)
$$

for all $v \in S$ and all $\alpha=1, \ldots, \nu$. Since there are only finitely many such tuples, we may subdivide $\mathscr{P}$ into finitely many subsets such that for each of them there exists one tuple $\left(\kappa_{v, \alpha}\right)$ for which (7.5.7) holds for all $P \in \mathscr{P}^{\prime}$. From this and (7.4), it follows that

$$
\frac{-\nu\left(E_{P}^{2}\right)}{[k: \mathbb{Q}]} \geq\left(\varepsilon-\nu \# S \varepsilon_{4}-\sum \kappa_{v, \alpha}\right) h(P) .
$$

By a similar finiteness argument, we can subdivide $\mathscr{P}$ further, so that for each subset $\mathscr{P}^{\prime}$, there exists a choice of points $Q_{v} \in \operatorname{Supp} D$ for all $v \in S$ and all $\alpha$ such that (7.5.4) holds for all $P \in \mathscr{P}^{\prime}$.

This leaves only condition (b).

In [V 4, 3.1.10], it is proved that there exists a constant $c_{12}=c_{12}(X)$ such that for all $P, P^{\prime} \in C(\bar{k})$,

$$
\frac{\left(E_{P} \cdot E_{P^{\prime}}\right)}{[k: \mathbb{Q}]} \leq h(P)+h\left(P^{\prime}\right)+\frac{\left(D_{P} \cdot D_{P^{\prime}}\right)}{[k: \mathbb{Q}]}+c_{12}
$$

using the decomposition

$$
E_{P} \equiv \frac{\omega_{X / B}}{2 g-2}+\left([k: \mathbb{Q}] h(P)-\frac{\left(\omega_{X / B}^{2}\right)}{(2 g-2)^{2}}\right) F_{P}+F_{P}^{\prime}+D_{P}
$$

from [V 4, 3.1.2]. Also, from [ $\mathrm{V} 4,3.1 .6]$, there exists a constant $c_{13}=c_{13}(X)$ such that

$$
\left({F_{P}^{\prime}}^{2}\right) \geq-[k: \mathbb{Q}] c_{13}+\frac{\left(\omega_{X / B}^{2}\right)}{(2 g-2)^{2}}
$$


and therefore it follows from [ $\mathrm{V} 4,3.1 .3]$ that

$$
\frac{\left(E_{P}^{2}\right)}{[k: \mathbb{Q}]} \geq 2 h(P)+\frac{\left(D_{P}^{2}\right)}{[k: \mathbb{Q}]}-c_{13}
$$

Thus, by (7.5.8),

$$
\begin{aligned}
-\frac{\left(D_{P}^{2}\right)}{[k: \mathbb{Q}]} & \geq 2\left(1+\frac{\varepsilon-\nu \# S \varepsilon_{4}-\sum \kappa_{v, \alpha}}{2 \nu}\right) h(P)-c_{13} \\
& \geq 2\left(1+\varepsilon_{5}+\varepsilon^{\prime}-\frac{\sum \kappa_{v, \alpha}}{2 \nu}\right) h(P)
\end{aligned}
$$

provided that $h(P) \geq c_{13} \nu /\left(\varepsilon-\nu \# S \varepsilon_{4}-2 \nu \varepsilon_{5}-2 \nu \varepsilon^{\prime}\right)$, which we now assume by setting

$$
h_{\min , 0}=\max \left(h_{0}, c_{13} \cdot \frac{\nu}{\varepsilon-\nu \# S \varepsilon_{4}-2 \nu \varepsilon_{5}-2 \nu \varepsilon^{\prime}}\right) .
$$

Lemma 7.5.10 [V 4, Lemma 3.1.12]. Fix $\theta \in(0, \pi]$. Then there exists a partitioning of the set of algebraic points $P_{i}$ into finitely many subsets such that, for all pairs $\left(P_{i}, P_{\ell}\right)$ both lying in the same such subset,

$$
-\left(D_{i} . D_{\ell}\right) \geq(\cos \theta) \sqrt{\left(-D_{i}^{2}\right)\left(-D_{\ell}^{2}\right)}
$$

Apply the lemma with

$$
\theta= \begin{cases}\arccos \left(\frac{1+\varepsilon^{\prime}-\sum \kappa_{v, \alpha} / 2 \nu}{1+\varepsilon_{5}+\varepsilon^{\prime}-\sum \kappa_{v, \alpha} / 2 \nu}\right), & \text { if } 1+\varepsilon^{\prime}-\sum \kappa_{v, \alpha} / 2 \nu>-\varepsilon_{5} / 2 \\ \pi, & \text { otherwise. }\end{cases}
$$

It follows that we may further refine the partitioning of $\mathscr{P}$ such that for all subsets $\mathscr{P}^{\prime}$ in the refinement and all points $P, P^{\prime} \in \mathscr{P}^{\prime}$ with $h(P) \geq h_{\min , 0}$ and $h\left(P^{\prime}\right) \geq h_{\min , 0}$,

$$
-\frac{\left(D_{P} \cdot D_{P^{\prime}}\right)}{[k: \mathbb{Q}]} \geq 2\left(1+\varepsilon^{\prime}-\frac{\sum \kappa_{v, \alpha}}{2 \nu}\right) \sqrt{h(P) h\left(P^{\prime}\right)} .
$$

Now for any distinct points $P_{1}, \ldots, P_{n} \in \mathscr{P}^{\prime}$ with $h\left(P_{i}\right) \geq h_{\min , 0}$, let

$$
s: E \rightarrow X \times_{B} \cdots \times_{B} X
$$

be the corresponding arithmetic curve, which is irreducible by the assumption that the fields $k(P)$ are all linearly disjoint over $k$. By (7.5.9) and (7.5.11),

$$
\frac{\operatorname{deg} s^{*} \Delta_{i \ell}^{\prime}}{\left[k\left(P_{1}, \ldots, P_{n}\right): \mathbb{Q}\right]} \leq-2\left(1+\varepsilon^{\prime}-\frac{\sum \kappa_{v, \alpha}}{2 \nu}\right) \sqrt{h\left(P_{i}\right) h\left(P_{\ell}\right)}+c_{12} .
$$

Note also that

$$
\frac{\operatorname{deg} s^{*} F_{i}}{\left[k\left(P_{1}, \ldots, P_{n}\right): \mathbb{Q}\right]}=\frac{\left(H_{i} \cdot F\right)}{\nu[k: \mathbb{Q}]}=h\left(P_{i}\right)
$$


Thus, for $\eta>0$ and tuples $(h)=\left(h_{1}, \ldots, h_{n}\right)$ satisfying (4.1) and (7.5.5),

$$
\begin{aligned}
\frac{\operatorname{deg} s^{*} Y_{x,(h)}}{\left[k\left(P_{1}, \ldots, P_{n}\right): \mathbb{Q}\right]} \leq & n\left(x(1+\eta)-(n-1)\left(1+\varepsilon^{\prime}-\frac{\sum \kappa_{v, \alpha}}{2 \nu}\right)\right) \\
& +c_{12} \sum_{i<\ell} \frac{1}{\sqrt{h_{i} h_{\ell}}} .
\end{aligned}
$$

\section{Estimates OF DERIVATIVES}

In this section we define certain "partial derivatives" of the section $\gamma$ constructed in $\S 6$, and estimate their norms at places in $S$. These estimates will then be used to obtain a lower bound for the index in $\S 9$.

These partial derivatives are defined as in $\S 6$, except that the definition takes place on a different model than $X$ or $W$. Let $\tilde{k}$ be a sufficiently large finite extension of $k$ such that for all (finite) places $v$ of $k$ for which $X$ has bad reduction, and all places $w$ of $\tilde{k}$ lying over $v$, the local field $\tilde{k}_{w}$ contains all extensions of $k_{v}$ of degree $\leq \nu$. Let $\tilde{\pi}: \tilde{X} \rightarrow \tilde{B}$ be a regular semistable model for $C$ over $\tilde{k}$. This model has the property that for all $n$-tuples of points $P_{1}, \ldots, P_{n} \in C^{n}(\bar{k})$ with $\left[k\left(P_{i}\right): k\right] \leq \nu$, the field $k^{\prime}:=\tilde{k}\left(P_{1}, \ldots, P_{n}\right)$ is unramified over $\tilde{k}$ at all places of bad reduction of $\tilde{X}$, and therefore the surface $X^{\prime}:=\widetilde{X} \times_{\tilde{B}} B^{\prime}$ (where $B^{\prime}$ is the arithmetic curve corresponding to $k^{\prime}$ ) is regular and semistable. Similar comments apply to the model $\widetilde{W}$ obtained by desingularizing $\widetilde{X} \times{ }_{\tilde{B}} \cdots \times_{\tilde{B}} \widetilde{X}$ and to $W^{\prime}:=\widetilde{W} \times_{\tilde{B}} B^{\prime}$.

By well-known properties of regular semistable models (e.g., [L 2, Chapter V, Theorem 5.1]), the rational map $\tilde{X} \rightarrow X$ is a morphism. If the choices made in the desingularization (2.4) of $\widetilde{W}$ are compatible with those made for $W$, then the map $\widetilde{W} \rightarrow W$ is also a morphism. Indeed, by the properties of the construction, it suffices to check this fact for the rational map $\widetilde{W}_{2} \rightarrow W_{2}$. This is easy to check by explicit computation, as follows. A node on a singular fibre on $X$ looks locally like $x y=\pi$, where $\pi$ is a uniformizer of the local ring $\mathscr{O}_{v}$ on $B$. Lifting to $\tilde{X}$, we obtain a singularity $x y=\tilde{\pi}^{e}$, where $e$ is the local index of ramification. Desingularizing produces a cover consisting of open affine subsets $U_{j}, j=0, \ldots, e-1$, where $x=x^{\prime} \tilde{\pi}^{j}$ and $y=y^{\prime} \tilde{\pi}^{e-j-1}$, so that again $x^{\prime} y^{\prime}=\tilde{\pi}$. Taking the product then gives singularities $x_{1}^{\prime} y_{1}^{\prime}=$ $\tilde{\pi}=x_{2}^{\prime} y_{2}^{\prime}$, where $x_{1}=x_{1}^{\prime} \tilde{\pi}^{j}, y_{1}=y_{1}^{\prime} \tilde{\pi}^{e-j-1}, x_{2}=x_{2}^{\prime} \tilde{\pi}^{k}$, and $y_{2}=y_{2}^{\prime} \tilde{\pi}^{e-k-1}$. These singularities are resolved by adjoining $w^{\prime}=x_{1}^{\prime} / x_{2}^{\prime}\left(=y_{2}^{\prime} / y_{1}^{\prime}\right)$ (and its inverse on a different open affine, which we may ignore by symmetry). We need to check that this open affine lies over some open affine on $W_{2}$ and that the regular functions on that open affine pull back to regular functions on $\widetilde{W}_{2}$. This amounts to checking that either $x_{1} / x_{2}$ or $x_{2} / x_{1}$ pulls back to a regular function. But if $j \geq k$, then $x_{1} / x_{2}=x_{1}^{\prime} \tilde{\pi}^{j} / x_{2}^{\prime} \tilde{\pi}^{k}=w^{\prime} \tilde{\pi}^{j-k}$ is regular; if $j<k$ then $x_{2} / x_{1}=x_{2}^{\prime} \tilde{\pi}^{k} / x_{1}^{\prime} \tilde{\pi}^{j}=x_{2}^{\prime} \tilde{\pi}^{k-j-1} y_{1}^{\prime}$ is regular. 
From the construction, it also follows from the above that the natural rational map $f: W^{\prime} \rightarrow W$ is a morphism. Then we may define the partial derivatives of $f^{*} \gamma$ along the image of the section $s^{\prime}: B^{\prime} \rightarrow W^{\prime}$ corresponding to $\left(P_{1}, \ldots, P_{n}\right)$. Let $(\ell)=\left(\ell_{1}, \ldots, \ell_{n}\right)$ be a tuple for which this derivative is defined and nonzero; then

$$
D_{(\ell)} f^{*} \gamma\left(P_{1}, \ldots, P_{n}\right) \in \Gamma\left(B^{\prime}, s^{\prime *} \mathscr{O}\left(d \cdot f^{*} Y_{x,(h)}-\ell_{1} \cdot \operatorname{pr}_{1}^{*} P_{1}-\cdots-\ell_{n} \cdot \operatorname{pr}_{n}^{*} P_{n}\right)\right) \text {. }
$$

Letting $f_{0}$ denote the natural morphism $X^{\prime} \rightarrow X$, we note that the relative dualizing sheaves behave well: $\omega_{X^{\prime} / B^{\prime}} \cong f_{0}^{*} \omega_{X / B}$. Then by the adjunction formula, (7.5.5), and the definition $d_{i}=d x / h_{i}$,

$$
\begin{gathered}
\frac{\operatorname{deg} s^{\prime *} \mathscr{O}\left(d \cdot f^{*} Y_{x,(h)}-\ell_{1} \cdot \operatorname{pr}_{1}^{*} P_{1}-\cdots-\ell_{n} \cdot \operatorname{pr}_{n}^{*} P_{n}\right)}{d\left[k^{\prime}: \mathbb{Q}\right]} \\
=\frac{\operatorname{deg} s^{*} \mathscr{O}\left(d Y_{x,(h)}\right)}{d\left[k^{\prime}: \mathbb{Q}\right]}+\sum_{i=1}^{n} \frac{\ell_{i}}{d} \cdot \frac{\operatorname{deg} s_{i}^{*} \omega_{X / B}}{\left[k\left(P_{i}\right): \mathbb{Q}\right]} \\
\leq \frac{\operatorname{deg} s^{*} \mathscr{O}\left(d Y_{x,(h)}\right)}{d\left[k^{\prime}: \mathbb{Q}\right]}+x(1+\eta)(2 g-2) \sum_{i=1}^{n} \frac{\ell_{i}}{d_{i}} .
\end{gathered}
$$

In the next section we will compare this bound for the above degree with the bound obtained from local bounds on the order of zero or pole of $D_{(\ell)} f^{*} \gamma\left(P_{1}, \ldots, P_{n}\right)$, which we shall compute next.

Remark. For the rest of the proof, we shall assume for simplicity that

$$
h\left(P_{1}\right)=\min \left(h\left(P_{1}\right), \ldots, h\left(P_{n}\right)\right),
$$

and similarly that $h_{1}=\min \left(h_{1}, \ldots, h_{n}\right)$.

First of all, for all non-archimedean places $w$ of $k^{\prime}$, the definition of this section immediately implies that it is regular. In particular, this gives a bound on $\left\|D_{(\ell)} f^{*} \gamma\left(P_{1}, \ldots, P_{n}\right)\right\|_{w}$, which will be used if $w$ lies over some $v \notin S$. But for other $w$ some stronger bounds are often possible. First, however, we compute a corresponding "worst case" bound for archimedean $w$.

Recall from $\S 6$ the definitions of $\rho$ and $U_{P}$ for $P \in C_{\sigma}$. By a compactness argument there exist constants $c_{14}=c_{14}(C)$ and $c_{15}=c_{15}(C)$ and local meromorphic sections $s_{1, P} \in \Gamma\left(U_{P}, \mathscr{O}(F)\right)$ and $s_{2, P_{1}, P_{2}} \in \Gamma\left(U_{P_{1}} \times U_{P_{2}}, \mathscr{O}\left(\Delta^{\prime}\right)\right)$ such that the bounds

$$
\frac{\left\|s_{1, P}\left(P^{\prime}\right)\right\|}{\left\|s_{1, P}(P)\right\|} \geq e^{-c_{14}} \quad \text { for all } P^{\prime} \in U_{P}
$$

and

$$
\frac{\left\|s_{2, P_{1}, P_{2}}\left(P_{1}^{\prime}, P_{2}^{\prime}\right)\right\|}{\left\|s_{2, P_{1}, P_{2}}\left(P_{1}, P_{2}\right)\right\|} \geq e^{-c_{15}} \quad \text { for all }\left(P_{1}^{\prime}, P_{2}^{\prime}\right) \in U_{P_{1}} \times U_{P_{2}}
$$

hold uniformly over $P \in C_{\infty}$ and $\left(P_{1}, P_{2}\right) \in(C \times C)_{\infty}$, respectively. Then for all points $\left(P_{1}, \ldots, P_{n}\right) \in\left(C^{n}\right)_{\infty}$ there exists a local holomorphic section $\gamma_{0}$ of 
$\mathscr{O}\left(d Y_{x,(h)}\right)$ on $U_{P_{1}} \times \cdots \times U_{P_{n}}$ such that

$$
\frac{\left\|\gamma_{0}(P)\right\|}{\left\|\gamma_{0}\left(P_{1}, \ldots, P_{n}\right)\right\|} \geq \exp \left(-c_{14} x d \sum_{i=1}^{n} \frac{1}{h_{i}}-c_{15} d \sum_{i<\ell} \frac{1}{\sqrt{h_{i} h_{\ell}}}\right)
$$

for all $P \in U_{P_{1}} \times \cdots \times U_{P_{n}}$. For future reference, we also have constants $c_{16}$ and $c_{17}$ such that

$$
\frac{\left\|\gamma_{0}(P)\right\|}{\left\|\gamma_{0}\left(P_{1}, \ldots, P_{n}\right)\right\|} \leq \exp \left(c_{16} x d \sum_{i=1}^{n} \frac{1}{h_{i}}+c_{17} d \sum_{i<\ell} \frac{1}{\sqrt{h_{i} h_{\ell}}}\right)
$$

for all $P \in U_{P_{1}} \times \cdots \times U_{P_{n}}$.

From Lemma 6.10 and (8.2) it follows that

$$
\begin{aligned}
& -\log \left\|D_{(\ell)} f^{*} \gamma\left(P_{1}, \ldots, P_{n}\right)\right\|_{w} \\
& \geq-\log \left\|f^{*} \gamma\right\|_{\text {sup }, w}+\sum_{i} \ell_{i} \log \rho-c_{14} x d \sum_{i} \frac{1}{h_{i}}-c_{15} d \sum_{i<\ell} \frac{1}{\sqrt{h_{i} h_{\ell}}} \\
& \quad \geq-\log \left\|f^{*} \gamma\right\|_{\text {sup }, w}-\frac{c_{18} d}{h_{1}}
\end{aligned}
$$

using the fact that $\ell_{i} \leq d_{i}$. Here $c_{18}=-n x \log \rho+c_{14} n x+c_{15} n(n-1) / 2$.

This estimate did not use (7.5.3). That condition will only be used for places $w$ of $k^{\prime}$ lying over places $v \in S$; moreover, it is not useful for all such places. To a place $w \mid v$ of $k^{\prime}$ we associate a tuple $(\alpha) \in[1, \ldots, \nu]^{n}$ by the condition that $\left(P_{1}, \ldots, P_{n}\right) \in C^{n}\left(k^{\prime}\right)$ coincides with the point $\left(P_{1}^{\left[\alpha_{1}\right]}, \ldots, P_{n}^{\left[\alpha_{n}\right]}\right) \in$ $C^{n}\left(\bar{k}_{v}\right)$ via the injection $k^{\prime} \hookrightarrow k_{w}^{\prime}$ at $w$ and the injection $k_{w}^{\prime} \hookrightarrow \bar{k}_{v}$. In general there may be many tuples associated to a given $w$, since there may be several injections $k_{w}^{\prime} \hookrightarrow \bar{k}_{v}$. But given a subset $\mathscr{S} \subseteq[1, \ldots, \nu]^{n}$, we have

$$
\sum_{w}^{\prime} \frac{\left[k_{w}^{\prime}: k_{v}\right]}{\left[k^{\prime}: k\right]} \geq \frac{\# \mathscr{S}}{\nu^{n}}
$$

where $\sum^{\prime}$ is the sum over all places corresponding to at least one tuple $(\alpha) \in$ $\mathscr{S}$.

The next estimate will hold only for places $w \mid v$ corresponding to at least one tuple $(\alpha)$ satisfying the condition

$$
\#\left\{i \mid \alpha_{i}=\beta\right\} \geq n\left(\frac{1}{\nu}-\varepsilon_{6}\right) \quad \text { for all } \beta=1, \ldots, \nu
$$

for some suitably chosen constant $\varepsilon_{6}>0$. The following lemma shows that this is the typical case.

Lemma 8.7 [W, Lemma 13]. Let $0<\varepsilon_{6}<1$. Then the number of tuples $(\alpha) \in$ $\mathbb{Z}^{n}$ with $1 \leq \alpha_{i} \leq \nu$ for $i=1, \ldots, n$ which do not satisfy (8.6) is at most $\nu^{n} / \varepsilon_{6}^{2} n$.

Lemma 8.8. Let $w$ be a place of $k^{\prime}$ lying over some place $v \in S$, and suppose thet $w$ corresponds to at least one tuple satisfying (8.6). Then for all $n$-tuples 
$\left(P_{1}, \ldots, P_{n}\right)$ of points, all of whose components lie in the same subset $\mathscr{P}^{\prime}$ as in Proposition 7.5, and for all suitable $(\ell)$,

$$
\begin{aligned}
& -\frac{1}{\left[k_{w}^{\prime}: k_{v}\right]} \log \left\|D_{(\ell)} f^{*} \gamma\left(P_{1}, \ldots, P_{n}\right)\right\|_{w} \\
& \geq d n x\left(\frac{1}{\nu}-\varepsilon_{6}\right)\left(\frac{\frac{1}{\nu}-\varepsilon_{6}+\frac{1}{n}}{2}-\varepsilon_{3}\right) \nu[k: \mathbb{Q}] \sum_{\alpha=1}^{\nu} \kappa_{v, \alpha} \\
& \quad-\nu[k: \mathbb{Q}] \sum_{i=1}^{n} \kappa_{v, \alpha_{i}} \ell_{i} h\left(P_{i}\right)-\frac{c_{19, v} d}{h_{1}}-\log \left\|f^{*} \gamma\right\|_{\text {sup }, w}-o(d)
\end{aligned}
$$

for some constant $c_{19, v}=c_{19, v}(X, D, n, x, \nu)$.

Proof. The proof splits up into cases.

Case I. The place $w$ is non-archimedean.

For $i=1, \ldots, n$ let $E_{i}$ and $E_{i}^{\prime}$ be the horizontal prime divisors on $X$ and $X^{\prime}$, respectively, corresponding to $P_{i}$, and let $E_{Q}$ and $E_{Q}^{\prime}$ be defined similarly for $Q \in \operatorname{Supp} D$. Then since the $Q$ 's are rational, $X$ is smooth in a neighborhood of $E_{Q}$ and therefore $X^{\prime} \cong X \times_{B} B^{\prime}$ near $E_{Q}^{\prime}$. Thus the local intersection numbers on $X^{\prime}$ at $w$ and on $X$ at $v$ are related, respectively, by the formula

$$
\left(E_{i}^{\prime} \cdot E_{Q}^{\prime}\right)_{X^{\prime}, w}=\left[k_{w}^{\prime}: k_{v}\right]\left(E_{i}^{\left[\alpha_{i}\right]} \cdot E_{Q}\right)_{X, v},
$$

where $E_{i}^{\left[\alpha_{i}\right]}$ is the local branch of $E_{i}$ corresponding to $P_{i}^{\left[\alpha_{i}\right]}$ and $(\alpha)$ is the tuple corresponding to $w$. Thus

$$
\left(E_{i}^{\prime} \cdot E_{Q}^{\prime}\right)_{X^{\prime}, w}=\left[k_{w}^{\prime}: k_{v}\right] g_{v}\left(P_{i}^{\left[\alpha_{i}\right]}, Q\right)
$$

by definition of $g_{v}$; hence by (7.5.3),

$$
\sum_{Q \in \operatorname{Supp} D}\left(E_{i}^{\prime} \cdot E_{Q}^{\prime}\right)_{w} \geq\left[k_{w}^{\prime}: k_{v}\right] \nu[k: \mathbb{Q}] \kappa_{v, \alpha_{i}} h\left(P_{i}\right) .
$$

Then there exists a constant $c_{20, v}=c_{20, v}(X, D) \geq 0$ such that, writing $Q_{i}=$ $Q_{v, \alpha_{i}}$,

$$
\left(E_{i}^{\prime} \cdot E_{Q_{i}}^{\prime}\right)_{w} \geq\left[k_{w}^{\prime}: k_{v}\right]\left(\nu[k: \mathbb{Q}] \kappa_{v, \alpha_{i}} h\left(P_{i}\right)-c_{20, v}\right) .
$$

Let $A \subseteq\{1, \ldots, \nu\}$ be the set of indices $\beta$ for which

$$
\nu[k: \mathbb{Q}] \kappa_{v, \beta} h\left(P_{1}\right)>c_{20, v} .
$$

For indices $i$ satisfying $\alpha_{i} \in A$ let $\xi_{i}$ be a locally defining equation for $E_{Q_{i}}^{\prime}$ over $v$; otherwise let $\xi_{i}$ be a locally defining equation for $E_{i}^{\prime}$. In both cases, let $\xi_{i}$ be identified with $\left(\operatorname{pr}_{i}^{\prime}\right)^{*} \xi_{i}$, where $\operatorname{pr}_{i}^{\prime}: W^{\prime} \rightarrow X^{\prime}$ is the projection onto the $i$ th factor. Then, the completed local ring of $W$ at $s^{\prime}(w)$ is

$$
\widehat{\mathscr{O}}_{W^{\prime}, s^{\prime}(w)} \cong \widehat{\mathscr{O}}_{B^{\prime}, w}\left[\left[\xi_{1}, \ldots, \xi_{n}\right]\right] \text {. }
$$


Letting $\gamma_{0}$ be some local section of $f^{*} \mathscr{O}\left(d Y_{x,(h)}\right)$ which is nonzero at $s^{\prime}(w)$, we have

$$
f^{*} \gamma=\gamma_{0} \sum_{(j) \geq 0} a_{(j)} \xi^{(j)}
$$

where $a_{(j)} \in \widehat{\mathscr{O}}_{B^{\prime}, w}$ for all $(j)$ (and in fact $\left|a_{(j)}\right| \leq\left\|f^{*} \gamma\right\|_{\text {sup }, w}$ ).

By construction, $\eta_{i}:=\xi_{i}-\xi_{i}\left(P_{i}\right)$ is a locally defining equation for $E_{i}^{\prime}$, for all $i$. Thus, $\widehat{\mathscr{O}}_{W^{\prime}, s^{\prime}(w)} \cong \widehat{\mathscr{O}}_{B^{\prime}, w}\left[\left[\eta_{1}, \ldots, \eta_{n}\right]\right]$ and so also

$$
\sum_{(j) \geq 0} a_{(j)} \xi^{(j)}=\sum_{(j) \geq 0} b_{(j)} \eta^{(j)}
$$

for some $b_{(j)} \in \widehat{\mathscr{O}}_{B^{\prime}, w}$. Moreover, by definition,

$$
\left\|D_{(\ell)} f^{*} \gamma\left(P_{1}, \ldots, P_{n}\right)\right\|_{w}=\left|b_{(\ell)}\right|_{w} .
$$

By Taylor's formula we may write

$$
b_{(\ell)}=\sum_{(j) \geq(\ell)} c_{(\ell),(j)} a_{(j)} \xi_{1}\left(P_{1}\right)^{j_{1}-\ell_{1}} \cdots \xi_{n}\left(P_{n}\right)^{j_{n}-\ell_{n}}
$$

for some integers $c_{(\ell),(j)} \in \mathbb{Z}$. By (8.8.1),

$$
\left|\xi_{i}\left(P_{i}\right)\right|_{w} \leq \exp \left(-\left[k_{w}^{\prime}: k_{v}\right]\left(\nu[k: \mathbb{Q}] \kappa_{v, \alpha_{i}} h\left(P_{i}\right)-c_{20, v}\right)\right) .
$$

Since $\xi_{i}\left(P_{i}\right)=0$ for all $i$ satisfying $\alpha_{i} \notin A$, we need only consider tuples (j) such that $j_{i}=\ell_{i}$ for those $i$. Also, combining the vanishing condition of Proposition 5.6(b) with (8.6), we need only consider tuples $(j)$ such that

$$
\sum_{\left\{i \mid \alpha_{i}=\beta\right\}} j_{i} h_{i} \geq d n x\left(\frac{1}{\nu}-\varepsilon_{6}\right)\left(\frac{\frac{1}{\nu}-\varepsilon_{6}+\frac{1}{n}}{2}-\varepsilon_{3}\right) \quad \text { for all } \beta \in A .
$$

For these tuples, (8.8.5) implies that

$$
\begin{aligned}
& -\log \left|\xi_{1}\left(P_{1}\right)^{j_{1}-\ell_{1}} \ldots \xi_{n}\left(P_{n}\right)^{j_{n}-\ell_{n}}\right|_{w} \\
& \quad \geq\left[k_{w}^{\prime}: k_{v}\right] \sum_{\left\{i \mid \alpha_{i} \in A\right\}}\left(j_{i}-\ell_{i}\right)\left(\nu[k: \mathbb{Q}] \kappa_{v, \alpha_{i}} h\left(P_{i}\right)-c_{20, v}\right) .
\end{aligned}
$$

But also $\left|\xi_{i}\left(P_{i}\right)\right|_{w}<1$, so we may reduce the $j_{i}$ so that equality holds in (8.8.6). This involves allowing fractional values of $j_{i}$. For such tuples,

$$
\sum_{\left\{i \mid \alpha_{i}=\beta\right\}} j_{i} \leq \sum_{\left\{i \mid \alpha_{i}=\beta\right\}} j_{i} \cdot \frac{h_{i}}{h_{1}} \leq \frac{d n x}{h_{1}}\left(\frac{1}{\nu}-\varepsilon_{6}\right)\left(\frac{\frac{1}{\nu}-\varepsilon_{6}+\frac{1}{n}}{2}-\varepsilon_{3}\right) .
$$


Thus

$$
\begin{aligned}
& -\frac{1}{\left[k_{w}^{\prime}: k_{v}\right]} \log \left|b_{(\ell)}\right|_{w} . \\
& \geq \operatorname{dnx}\left(\frac{1}{\nu}-\varepsilon_{6}\right)\left(\frac{\frac{1}{\nu}-\varepsilon_{6}+\frac{1}{n}}{2}-\varepsilon_{3}\right)\left(\nu[k: \mathbb{Q}] \sum_{\alpha \in A} \kappa_{v, \alpha}-\frac{(\# A) c_{20, v}}{h_{1}}\right) \\
& \quad-\nu[k: \mathbb{Q}] \sum_{\left\{i \mid \alpha_{i} \in A\right\}} \kappa_{v, \alpha_{i}} \ell_{i} h\left(P_{i}\right)-\log \left\|f^{*} \gamma\right\|_{\text {sup }, w} \\
& \geq \\
& \quad d n x\left(\frac{1}{\nu}-\varepsilon_{6}\right)\left(\frac{\frac{1}{\nu}-\varepsilon_{6}+\frac{1}{n}}{2}-\varepsilon_{3}\right)\left(\nu[k: \mathbb{Q}] \sum_{\alpha=1}^{\nu} \kappa_{v, \alpha}-\frac{\nu(1+\eta) c_{20, v}}{h_{1}}\right) \\
& \quad-\nu[k: \mathbb{Q}] \sum_{i=1}^{n} \kappa_{v, \alpha_{i}} \ell_{i} h\left(P_{i}\right)-\log \left\|f^{*} \gamma\right\|_{\text {sup }, w},
\end{aligned}
$$

using (8.8.2) and (7.5.5) for the second inequality. The result then follows from (8.8.3), with

$$
c_{19, v}=n x \nu(1+\eta)\left(\frac{1}{\nu}-\varepsilon_{6}\right)\left(\frac{\frac{1}{\nu}-\varepsilon_{6}+\frac{1}{n}}{2}-\varepsilon_{3}\right) c_{20, v} .
$$

Case II. The place $w$ is archimedean, corresponding to $\sigma: k^{\prime} \hookrightarrow \mathbb{C}$. The proof in this case is similar to the non-archimedean case, except that we shall again use $\rho$ and $U_{P}$ for $P \in C_{\sigma}$, as in (8.2). Recall that $C_{\sigma}$ denotes the complex curve (i.e., Riemann surface) $C \times{ }_{\sigma} \mathbb{C}$ and, for a rational point $P, P_{\sigma}$ denotes the corresponding point on $C_{\sigma}$.

There exists a constant $c_{21, v}=c_{21, v}(C, D) \geq 1$ such that if $P \in C_{\sigma}$ and $G_{\sigma}(P, D) \leq \rho / c_{21, v}$, then $P \in U_{Q, \sigma}$ for some $Q \in \operatorname{Supp} D$ and

$$
\left|z_{Q}(P)\right| \leq c_{21, v} G_{\sigma}(P, D) \text {. }
$$

Then let $A \subseteq\{1, \ldots, \nu\}$ be defined as the set of indices $\beta$ for which

$$
\nu[k: \mathbb{Q}] \kappa_{v, \beta} h\left(P_{1}\right) \geq \log \left(3 c_{21, v} / \rho\right) .
$$

For indices $i$ satisfying $\alpha_{i} \in A$ let $Q_{i}=\left(Q_{v, \alpha_{i}}\right)_{\sigma}, U_{i}=U_{Q_{i}}$, and $z_{i}=z_{Q_{i}}$; for these values of $i$,

$$
\left|z_{i}\left(P_{i, \sigma}\right)\right| \leq c_{21, v} \exp \left(-\nu[k: \mathbb{Q}] \kappa_{v, \alpha_{i}} h\left(P_{i}\right)\right) \leq \frac{\rho}{3} .
$$

For the remaining values of $i$, let $Q_{i}=P_{i, \sigma}, U_{i}=U_{Q_{i}}$, and $z_{i}=z_{Q_{i}}$. Let $\gamma_{0}$ be the local section as in (8.2); then

$$
f^{*} \gamma=\gamma_{0} \sum_{(j) \geq 0} a_{(j)} z^{(j)}=\gamma_{0} \sum_{(j) \geq 0} b_{(j)}\left(z_{1}-z_{1}\left(P_{1, \sigma}\right)\right)^{j_{1}} \cdots\left(z_{n}-z_{n}\left(P_{n, \sigma}\right)\right)^{j_{n}}
$$

as before. By Lemma 6.9,

$$
\left\|D_{(\ell)} f^{*} \gamma\left(P_{1}, \ldots, P_{n}\right)\right\|_{\sigma} \leq\left\|\gamma_{0}\left(P_{1, \sigma}, \ldots, P_{n, \sigma}\right)\right\|\left|b_{(\ell)}\right| .
$$

Also, by the Cauchy integral formula (6.10.2),

$$
\left|a_{(j)}\right| \leq \frac{\left\|f^{*} \gamma\right\|_{\text {sup }, \sigma}}{\inf _{P \in U_{1} \times \cdots \times U_{n}}\left\|\gamma_{0}(P)\right\|} \rho^{-j_{1}-\cdots-j_{n}}
$$


Note that in (8.8.4) the constants $c_{(\ell),(j)}$ are bounded:

$$
c_{(\ell),(j)}=\left(\begin{array}{l}
j_{1} \\
\ell_{1}
\end{array}\right) \cdots\left(\begin{array}{l}
j_{n} \\
\ell_{n}
\end{array}\right) \leq 2^{j_{1}+\cdots+j_{n}} .
$$

Then $\left|b_{(\ell)}\right|$ is bounded by the sum

$$
\left|b_{(\ell)}\right| \leq \sum_{(j) \geq(\ell)} 2^{j_{1}+\cdots+j_{n}}\left|a_{(j)}\right|\left|z_{1}\left(P_{1, \sigma}\right)\right|^{j_{1}-\ell_{1}} \cdots\left|z_{n}\left(P_{n, \sigma}\right)\right|^{j_{n}-\ell_{n}} .
$$

Thus by (8.8.9) and (8.8.8),

$$
\begin{aligned}
& \frac{\inf _{P \in U_{1} \times \cdots \times U_{n}}\left\|\gamma_{0}(P)\right\|}{\left\|f^{*} \gamma\right\|_{\text {sup }, \sigma}}\left|b_{(\ell)}\right| \\
& \quad \leq\left(\frac{2}{\rho}\right)^{\ell_{1}+\cdots+\ell_{n}} \sum_{(j) \geq(\ell)} \exp \left(-\sum_{i=1}^{n}\left(j_{i}-\ell_{i}\right)\left(\nu[k: \mathbb{Q}] \kappa_{v, \alpha_{i}} h\left(P_{i}\right)-\log \left(2 c_{21, v} / \rho\right)\right)\right) .
\end{aligned}
$$

As in the non-archimedean case, the second sum can be restricted to the tuples $(j)$ which satisfy (8.8.6) and which also satisfy $j_{i}=\ell_{i}$ for all $i$ such that $\alpha_{i} \notin A$. This set can be covered by finitely many translated quadrants. By (8.8.7) and a geometric series argument, the sum over all $\left(j^{\prime}\right)$ in a translated quadrant with origin $(j)$ is bounded by

$$
3^{n}(2 / \rho)^{\ell_{1}+\cdots+\ell_{n}} \exp \left(-\sum_{i=1}^{n}\left(j_{i}-\ell_{i}\right)\left(\nu[k: \mathbb{Q}] \kappa_{v, \alpha_{i}} h\left(P_{i}\right)-\log \left(2 c_{21, v} / \rho\right)\right)\right) .
$$

Since $\ell_{i} \leq d_{i}$ for all $i$, the number of such quadrants can be taken less than $e^{o(d)}$.

Thus, as in the previous case (but using (8.8.7) instead of (8.8.2)),

$$
\begin{aligned}
-\log \left|b_{(\ell)}\right| & \\
\geq & d n x\left(\frac{1}{\nu}-\varepsilon_{6}\right) \\
\quad \times & \left(\frac{\frac{1}{\nu}-\varepsilon_{6}+\frac{1}{n}}{2}-\varepsilon_{3}\right)\left(\nu[k: \mathbb{Q}] \sum_{\alpha=1}^{\nu} \kappa_{v, \alpha}-\frac{\nu(1+\eta) \log \left(3 c_{21, v} / \rho\right)}{h_{1}}\right) \\
& -\nu[k: \mathbb{Q}] \sum_{i=1}^{n} \kappa_{v, \alpha_{i}} \ell_{i} h\left(P_{i}\right)-\sum_{i=1}^{n} \ell_{i} \log (2 / \rho) \\
& -\log \left\|f^{*} \gamma\right\|_{\text {sup }, \sigma}+\log \inf _{P \in U_{1} \times \cdots \times U_{n}}\left\|\gamma_{0}(P)\right\|-o(d) .
\end{aligned}
$$

By (8.2) and (8.3) the desired bound then follows, with

$$
\begin{aligned}
c_{19, v}= & n x \nu(1+\eta)\left(\frac{1}{\nu}-\varepsilon_{6}\right)\left(\frac{\frac{1}{\nu}-\varepsilon_{6}+\frac{1}{n}}{2}-\varepsilon_{3}\right) \log \left(3 c_{21, v} / \rho\right) \\
& +n x\left(\log (2 / \rho)+c_{14}+c_{16}\right)+n(n-1)\left(c_{15}+c_{17}\right) / 2 .
\end{aligned}
$$




\section{A LOWER BOUND FOR THE INDEX}

We are now ready to prove a lower bound for the index of $\gamma$ at $\left(P_{1}, \ldots, P_{n}\right)$.

Proposition 9.1. For any point $\left(P_{1}, \ldots, P_{n}\right)$ as in Proposition 7.5 the index

$$
t=t\left(\gamma,\left(P_{1}, \ldots, P_{n}\right), d_{1}, \ldots, d_{n}\right)
$$

satisfies the inequality

$$
\frac{t}{n} \geq \frac{A-c_{22} / h_{1}}{\left(1+\varepsilon_{1}\right)(1+\eta)\left(2 g-2+\nu \# S\left(\operatorname{deg} D+2 \varepsilon_{4}\right)\right)}-o(1),
$$

where

$$
\begin{aligned}
A=1 & +\varepsilon^{\prime}-\left(1+\varepsilon_{1}\right)(1+\eta) \\
& +\frac{\sum \kappa_{v, \alpha}}{2 \nu}\left(\left(1+\varepsilon_{1}\right)\left(1-\frac{1}{\varepsilon_{6}^{2} n}\right)\left(1-\varepsilon_{6} \nu\right)\left(1-\varepsilon_{6} \nu+\frac{\nu}{n}-2 \varepsilon_{3} \nu\right)-1\right)
\end{aligned}
$$

and $c_{22}$ is independent of $P_{1}, \ldots, P_{n}, h_{1}, \ldots, h_{n}$, and $d$.

Proof. First, we sum the bounds (8.4) and (8.8) over all places $w$ of $k^{\prime}$ lying over all $v \in S$. Over remaining places of $k^{\prime}, D_{(\ell)} f^{*} \gamma$ is integral; therefore this sum gives a lower bound on the degree of $D_{(\ell)} f^{*} \gamma\left(P_{1}, \ldots, P_{n}\right)$. Of course the stronger bound (8.8) is not valid for all $w$ lying over a given place $v$, but by (8.5) and Lemma 8.7 the weighted proportion of $w$ for which (8.8) can be used is at least $\left(1-1 / \varepsilon_{6}^{2} n\right)$. Thus using (6.12.1) and (7.5.5), we have

$$
\begin{aligned}
\frac{\operatorname{deg} D_{(\ell)} f^{*} \gamma\left(P_{1}, \ldots, P_{n}\right)}{d\left[k^{\prime}: \mathbb{Q}\right]} \\
\geq n x\left(1-\frac{1}{\varepsilon_{6}^{2} n}\right)\left(\frac{1}{\nu}-\varepsilon_{6}\right)\left(\frac{\frac{1}{\nu}-\varepsilon_{6}+\frac{1}{n}}{2}-\varepsilon_{3}\right) \nu \sum \kappa_{v, \alpha} \\
\quad-x(1+\eta) \sum \kappa_{v, \alpha} \sum_{i=1}^{n} \frac{\ell_{i}}{d_{i}}-\frac{c_{23}}{h_{1}}-o(1),
\end{aligned}
$$

where

$$
c_{23}=\left(1-\frac{1}{\varepsilon_{6}^{2} n}\right) \sum_{v \in S} \frac{c_{19, v}}{[k: \mathbb{Q}]}+\frac{c_{18}}{\varepsilon_{6}^{2} n}+c_{4} n .
$$

But also (8.1) and (7.5.6) combine to give

$$
\begin{aligned}
\frac{\operatorname{deg} D_{(\ell)} f^{*} \gamma\left(P_{1}, \ldots, P_{n}\right)}{d\left[k^{\prime}: \mathbb{Q}\right]} \leq n & \left(x(1+\eta)-(n-1)\left(1+\varepsilon^{\prime}-\frac{\sum \kappa_{v, \alpha}}{2 \nu}\right)\right) \\
& +x(1+\eta)(2 g-2) \sum_{i=1}^{n} \frac{\ell_{i}}{d_{i}}+c_{12} \sum_{i<\ell} \frac{1}{\sqrt{h_{i} h_{\ell}}} .
\end{aligned}
$$

These inequalities are valid for any tuple $(\ell)$ for which $D_{(\ell)} f^{*} \gamma\left(P_{1}, \ldots, P_{n}\right)$ is defined and nonzero. In particular, they hold for a tuple $(\ell)$ for which 


$$
t=\sum_{i=1}^{n} \frac{\ell_{i}}{d_{i}}
$$

For such a tuple $(\ell)$, combining the two inequalities gives

$$
t \geq \frac{A^{\prime}-n(n-1) c_{22} / h_{1}}{x(1+\eta)\left(2 g-2+\sum \kappa_{v, \alpha}\right)}-o(1),
$$

where

$$
\begin{aligned}
A^{\prime}=- & n\left(x(1+\eta)-(n-1)\left(1+\varepsilon^{\prime}-\frac{\sum \kappa_{v, \alpha}}{2 \nu}\right)\right) \\
& +n x\left(1-\frac{1}{\varepsilon_{6}^{2} n}\right)\left(\frac{1}{\nu}-\varepsilon_{6}\right)\left(\frac{\frac{1}{\nu}-\varepsilon_{6}+\frac{1}{n}}{2}-\varepsilon_{3}\right) \nu \sum \kappa_{v, \alpha}
\end{aligned}
$$

and $c_{22}=c_{23} / n(n-1)+c_{12}$. Substituting $x=(n-1)\left(1+\varepsilon_{1}\right)$ and simplifying then gives $(9.1 .1)$.

\section{Roth's LEMMA}

This section sets up the framework for applying Roth's lemma. The version to be used is the following.

Definition 10.1. Let $Q$ be a polynomial with algebraic coefficients. Then the height of $Q$, denoted $h(Q)$, is the height of its vector of coefficients (Definition $6.1)$.

Lemma 10.2 (Roth, [R]; see also [B]). Let $Q\left(\zeta_{1}, \ldots, \zeta_{n}\right) \not \equiv 0$ be a polynomial in $n$ variables, of degree at most $r_{i}$ in $\zeta_{i}$, with algebraic coefficients. Let $\xi=$ $\left(\xi_{1}, \ldots, \xi_{n}\right)$ be an algebraic point, and let $t^{\prime}=t\left(Q, \xi, r_{1}, \ldots, r_{n}\right)$ be the index of $Q$ at $\xi$. Suppose that $\varepsilon_{8}>0$ is such that

$$
\frac{r_{i+1}}{r_{i}} \leq \varepsilon_{8}^{2^{n-1}}, \quad i=1, \ldots, n-1,
$$

and

$$
r_{i} h\left(\xi_{i}\right) \geq \varepsilon_{8}^{-2^{n-1}}\left(h(Q)+2 n r_{1}\right), \quad i=1, \ldots, n
$$

Then

$$
\frac{t^{\prime}}{n} \leq 2 \varepsilon_{8}
$$
$C^{n}$ :

The goal of this section is to translate this lemma into a similar statement on

Lemma 10.3. There exists a constant $c_{25}=c_{25}(C, n, x)$ with the following property. Let $\gamma$ be a section of $\mathscr{O}\left(d Y_{x,(h)}\right)$ with height

$$
h(\gamma):=\frac{1}{[k: \mathbb{Q}]} \sum_{\sigma \in B_{\infty}} \log \|\gamma\|_{\text {sup }, \sigma} .
$$

Let $\left(P_{1}, \ldots, P_{n}\right)$ be an algebraic point on $C^{n}$, and let

$$
t=t\left(\gamma,\left(P_{1}, \ldots, P_{n}\right), d_{1}, \ldots, d_{n}\right)
$$


be the index of $\gamma$ at $\left(P_{1}, \ldots, P_{n}\right)$. Suppose that $\varepsilon_{8}>0$ is such that

$$
\frac{h_{i+1}}{h_{i}} \geq \varepsilon_{8}^{-2^{n-1}}, \quad i=1, \ldots, n-1,
$$

and

(10.3.2)

$$
d x \cdot \frac{h\left(P_{i}\right)-c_{24}}{h_{i}} \geq \varepsilon_{8}^{-2^{n-1}}\left(h(\gamma)+\frac{\left(c_{25}+2 n x / N\right) d}{h_{1}}\right), \quad i=1, \ldots, n .
$$

Then

$$
\frac{t}{n} \leq 2 N^{n-1} \varepsilon_{8} .
$$

Proof. We may regard the polynomial $Q$ in Roth's lemma as a section of the line sheaf $\mathscr{O}\left(r_{1}, \ldots, r_{n}\right)$ on $\left(\mathbb{P}^{1}\right)^{n}$. To relate this section with $\gamma$, we take the norm from $C^{n}$ to $\left(\mathbb{P}^{1}\right)^{n}$ : by Assumption 6.6(a), the map from $C$ to $\mathbb{P}^{1}$ defined by the rational function $x_{1} / x_{0}$ is a morphism, and $\phi_{N F}^{*} \mathscr{O}(1)$ coincides with the pull-back of $\mathscr{O}(1)$ from $\mathbb{P}^{1}$. Therefore there exists a constant $c_{24}=c_{24}\left(\phi_{N F}\right)$ such that for all $P \in C(\bar{k})$,

$$
h\left(\left(x_{1} / x_{0}\right)(P)\right) \geq N\left(h(P)-c_{24}\right) .
$$

The morphism $C \rightarrow \mathbb{P}^{1}$ has degree $N$, so the morphism $C^{n} \rightarrow\left(\mathbb{P}^{1}\right)^{n}$ has degree $N^{n}$. Via the latter map, we will take the norm to $\left(\mathbb{P}^{1}\right)^{n}$ of $\gamma$, giving a section of $\mathscr{O}\left(r_{1}, \ldots, r_{n}\right)$ (i.e., a polynomial $Q$ as in Lemma 10.2), where

$$
r_{i}=N^{n-1} d_{i}, \quad i=1, \ldots, n .
$$

Let $\left(\xi_{1}, \ldots, \xi_{n}\right)$ be the image of $\left(P_{1}, \ldots, P_{n}\right)$ in $\left(\mathbb{P}^{1}\right)^{n} ;$ then

$$
t^{\prime} \geq \frac{t}{N^{n-1}}
$$

Therefore, in order to pull back Roth's lemma to $C^{n}$, it only remains to compare the heights $h(Q)$ and $h(\gamma)$.

To do this, we consider the definition of the norm in more detail. Let $K^{*}$ be a finite extension of $K(X)$ which is normal over $K\left(\mathbb{P}_{B}^{1}\right)$. There exists a model $X^{*}$ for $K^{*}$ such that the rational maps $X^{*} \rightarrow X$ corresponding to all injections $K(X) \hookrightarrow K^{*}$ over $K\left(\mathbb{P}_{B}^{1}\right)$ are morphisms. The product of the pullbacks of $\mathscr{O}(F)$ via these morphisms is a line sheaf on $X^{*}$ which is isomorphic to the pull-back of $\mathscr{O}(N)$ on $P_{B}^{1}$ along the generic fibre. This isomorphism amounts to tensoring with some section of some line sheaf on $X^{*}$ whose divisor is supported only along components of fibres over $B$. Any section of $\mathscr{O}(d F)$ can then be pushed down to a section of $\mathscr{O}(d N)$ which is global on $\mathbb{P}_{B}^{1}$ up to denominators which are annihilated by an integer bounded by $e^{O(d)}$. Similar comments apply to $\mathscr{O}\left(\Delta^{\prime}\right)$ on $W_{2}$. We then combine these to determine that the norm of $\gamma$ is a multihomogeneous polynomial with degrees given by (10.3.5), 
such that the denominators of coefficients are annihilated by an integer bounded by $\exp \left(c_{26} d / h_{1}\right)$, for some constant $c_{26}=c_{26}(X, n, x)$. Likewise

$$
\|\operatorname{Norm}(\gamma)\|_{\text {sup }, \sigma} \leq\|\gamma\|_{\text {sup }, \sigma}^{N^{n}} \exp \left(c_{27} d / h_{1}\right), \quad \text { for all } \sigma \in B_{\infty},
$$

where $c_{27}=c_{27}(C, n, x)$ and the metric on the left is derived from the FubiniStudy metric on $\mathbb{P}^{1}$. A simple application of the Cauchy integral formula to the polydisc $\mathbb{D}^{n} \subseteq \mathbb{P}^{1}(\mathbb{C})^{n}$ gives the inequality

$$
h(Q) \leq \frac{1}{[k: \mathbb{Q}]} \sum_{\sigma \in B_{\infty}} \log \|\operatorname{Norm}(\gamma)\|_{\text {sup }, \sigma}+\log m,
$$

where $Q$ is the polynomial corresponding to the norm of $\gamma$ and $m$ is an integer annihilating the denominators of $Q$. Thus

$$
h(Q) \leq N^{n}\left(h(\gamma)+\frac{c_{25} d}{h_{1}}\right)
$$

where $c_{25}=\left(c_{26}+c_{27}\right) / N^{n}$.

To finish the proof, we now apply Roth's lemma. By (10.3.1) and (10.3.5), condition (10.2.1) holds; by (10.3.2), (10.3.4), (10.3.5), and (10.3.7), condition (10.2.2) holds. Then, by (10.2.3) and (10.3.6), the conclusion (10.3.3) follows.

\section{Proof of Theorem 0.1}

First choose $\varepsilon^{\prime}, \varepsilon_{1}, \varepsilon_{3}, \varepsilon_{4}$, and $\varepsilon_{6}$, all positive real numbers, such that $\varepsilon_{1}$ is rational, such that Propositions 3.2 and 5.6 hold, such that $\nu \# S \varepsilon_{4}+2 \nu \varepsilon^{\prime}<\varepsilon$ (from (7.5.1)), and such that

$$
\varepsilon^{\prime}-\varepsilon_{1}+\frac{\# S\left(\operatorname{deg} D+2 \varepsilon_{4}\right)}{2} \min \left(0,\left(1+\varepsilon_{1}\right)\left(1-\varepsilon_{6} \nu\right)\left(1-\varepsilon_{6} \nu-2 \varepsilon_{3} \nu\right)-1\right)>0
$$

Then choose $n$ sufficiently large so that $n>n_{0}$ (as in Proposition 5.6), such that $n>1 / \varepsilon_{6}^{2}$, and such that

$$
\varepsilon^{\prime}-\varepsilon_{1}+\frac{\# S\left(\operatorname{deg} D+2 \varepsilon_{4}\right)}{2 \nu} M>0
$$

where

$$
M=\min \left(0,\left(1+\varepsilon_{1}\right)\left(1-\frac{1}{\varepsilon_{6}^{2} n}\right)\left(1-\varepsilon_{6} \nu\right)\left(1-\varepsilon_{6} \nu+\frac{\nu}{n}-2 \varepsilon_{3} \nu\right)-1\right) .
$$

Then choose $\varepsilon_{7}>0$ and $\varepsilon_{8}>0$ such that

$$
\frac{\varepsilon^{\prime}-\varepsilon_{1}+\frac{\# S\left(\operatorname{deg} D+2 \varepsilon_{4}\right)}{2 \nu} M-\varepsilon_{7}}{\left(1+\varepsilon_{1}\right)\left(2 g-2+\nu \# S\left(\operatorname{deg} D+2 \varepsilon_{4}\right)\right)}>2 n N^{n-1} \varepsilon_{8}
$$

Assume that the theorem is false. Then one of the subsets $\mathscr{P}$ of Proposition 7.5 is infinite. Let

$$
h_{\min }=\max \left(h_{\min , 0}, \frac{c_{22}}{\varepsilon_{7}}, c_{24}+\varepsilon_{8}^{-2^{n-1}}\left(\frac{c_{4} n+c_{25}}{x}+\frac{2 n}{N}\right)\right) .
$$


There exist points $P_{1}, \ldots, P_{n} \in \mathscr{P}$ satisfying (4.4.1) and

$$
\frac{h\left(P_{i+1}\right)}{h\left(P_{i}\right)},>\varepsilon_{8}^{-2^{n-1}} \quad \text { for all } i=1, \ldots, n-1,
$$

and such that $h\left(P_{1}\right)>h_{\text {min }}$. For all sufficiently small $\eta>0$, we can then choose a tuple $\left(h_{1}, \ldots, h_{n}\right)$ satisfying $(4.1),(4.4 .1),(7.5 .5)$, and the condition $h_{1}>h_{\min }$. In particular it follows that $c_{22} / h_{1}<\varepsilon_{7}$ and therefore (for all sufficiently small $\eta>0$ and all sufficiently large and divisible $d$, depending on $\eta$ ), the section $\gamma$ determined by all these choices satisfies the condition

$$
\frac{t}{n}>2 N^{n-1} \varepsilon_{8}
$$

where $t$ is the index of $\gamma$ at $\left(P_{1}, \ldots, P_{n}\right)$, by Proposition 9.1 and (7.5.2). This contradicts Roth's lemma 10.3. Indeed, (11.3) and (7.5.5) imply (10.3.1) for all sufficiently small $\eta ;(7.5 .1)$, the last part of (11.2), (6.12.1), and (7.5.5) imply (10.3.2) for $\eta$ small enough. Then (10.3.3) holds, contradicting (11.4). Thus Theorem 0.1 is proved.

\section{REFERENCES}

[B] E. Bombieri, The Mordell conjecture revisited, Ann. Scuola Norm. Sup. Pisa Cl. Sci. (4) 17 (1990), 615-640.

[B-V] E. Bombieri and J. Vaaler, On Siegel's lemma, Invent. Math. 73 (1983), 11-32; addendum, ibid. 75 (1984), 377.

[F 1] G. Faltings, Endlichkeitssätze für abelsche Varietäten über Zahlkörpern, Invent. Math. 73 (1983), 349-366; corrigendum, ibid. 75 (1984), 381.

[F 2] _ Diophantine approximation on abelian varieties, Ann. of Math. (2) 133 (1991), 549576.

[L 1] S. Lang, Fundamentals of diophantine geometry, Springer-Verlag, New York, 1983.

[L 2] _ Introduction to Arakelov theory, Springer-Verlag, New York, 1988.

[M] D. Mumford, A remark on Mordell's conjecture, Amer. J. Math. 87 (1965), 1007-1016.

[R] K. F. Roth, Rational approximations to algebraic numbers, Mathematika 2 (1955), 1-20; corrigendum, ibid. 2 (1955), 168.

[Sil] J. H. Silverman, Rational points on symmetric products of a curve, preprint 1989.

[T] A. Thue, Über Annäherungswerte algebraischer Zahlen, J. Reine Angew. Math. 135 (1909), 284-305.

[V 1] P. Vojta, Diophantine approximations and value distribution theory, Lecture Notes in Mathematics, vol. 1239, Springer-Verlag, New York, 1987.

[V 2] _ On algebraic points on curves, Compositio Math. 78 (1991), 29-36.

[V 3] _ Mordell's conjecture over function fields, Invent. Math. 98 (1989), 115-138.

[V 4] _ Siegel's theorem in the compact case, Ann. of Math. (2) 133 (1991), 509-548.

[V 5] Arithmetic discriminants and quadratic points on curves, Arithmetic Algebraic Geometry, Texel 1989 (G. van der Geer, F. Oort, and J. H. M. Steenbrink, eds.), Prog. in Math., vol. 89, Birkhäuser, Boston, 1991, pp. 359-376.

[V 6] - Applications of arithmetic algebraic geometry to diophantine approximations, Proceedings of CIME Conference, Trento, 1991, Lecture Notes in Mathematics, Springer-Verlag, Heidelberg (to appear).

[W] E. Wirsing, On approximation of algebraic numbers by algebraic numbers of bounded degree, 1969 Number Theory Institute, Proc. Sympos. Pure Math., vol. 20, Amer. Math. Soc., Providence, R.I., 1971, pp. 213-247.

Department of Mathematics, University of California, Berkeley, California 94720 E-mail address: vojta@math.berkeley.edu 